\title{
ZERO DISSIPATION LIMIT TO RAREFACTION WAVE WITH VACUUM FOR ONE-DIMENSIONAL FULL COMPRESSIBLE NAVIER-STOKES EQUATIONS*
}

\author{
MING-JIE $\mathrm{LI}^{\dagger}$ AND TENG WANG $\ddagger$
}

\begin{abstract}
We study the zero dissipation limit of the full compressible Navier-Stokes equations to a rarefaction wave which connects to vacuum at one side. It is shown that there exists a family of smooth solutions to the full compressible Navier-Stokes equations converging to the rarefaction wave with vacuum away from the initial layers at a uniform rate as the viscosity and the heat conduction coefficient tend to zero. Our method of proof consists of a scaling argument and elementary energy analysis based on the underlying wave structure.
\end{abstract}

Key words. Compressible fluid, Navier-Stokes equations, rarefaction wave, vacuum, zero dissipation limit.

AMS subject classifications. 35L60, 35L65, 76N15.

\section{Introduction}

This paper is a sequel of our previous work [3] on studying the asymptotic equivalence between the solutions of a one-dimensional model system for the compressible Navier-Stokes equations and those of the compressible Euler system with vacuum. The previous paper is concerned isentropic case, while the present one treats the compressible Navier-Stokes equations with heat-conduction. The one-dimensional full compressible Navier-Stokes equations in Eulerian coordinates read

$$
\left\{\begin{array}{l}
\rho_{t}+(\rho u)_{x}=0, \\
(\rho u)_{t}+\left(\rho u^{2}+p\right)_{x}=\left(\epsilon u_{x}\right)_{x}, \\
{\left[\rho\left(e+\frac{u^{2}}{2}\right)\right]_{t}+\left[\rho u\left(e+\frac{u^{2}}{2}\right)+u p\right]_{x}=\left(\kappa \theta_{x}+\epsilon u u_{x}\right)_{x},}
\end{array}\right.
$$

for $x \in \mathbf{R}=(-\infty,+\infty), t>0$, where $\rho \geq 0, u(x, t), p(x, t), e(x, t) \geq 0$, and $\theta(x, t) \geq 0$ are the mass density, fluid velocity, pressure, internal energy, and absolute temperature respectively, while the positive constants $\epsilon$ and $\kappa$ denote the viscosity and heat conductivity coefficient respectively. Here we study the ideal polytropic gas so that $p$ and $e$ are given by the state equations

$$
p=R \rho \theta=A \rho^{\gamma} \exp \left(\frac{\gamma-1}{R} S\right), \quad e=\frac{R}{\gamma-1} \theta+\text { const. },
$$

where $S$ is the entropy, $\gamma>1$ is the adiabatic exponent, and $A$ and $R$ are positive constants. Due to the second law of thermodynamics,

$$
d e=\theta d S+\frac{p}{\rho^{2}} d \rho,
$$

the following entropy equation can be derived from (1.1):

$$
(\rho S)_{t}+(\rho u S)_{x}=\left(\kappa \frac{\theta_{x}}{\theta}\right)_{x}+\kappa \frac{\left(\theta_{x}\right)^{2}}{\theta^{2}}+\epsilon \frac{\left(u_{x}\right)^{2}}{\theta} .
$$

*Received: May 29, 2013; accepted (in revised form): August 15, 2013. Communicated by Yaguang Wang.

${ }^{\dagger}$ College of Science, Minzu University of China, Beijing 100081, P.R. China (lmjmath@gmail.com).

${ }^{\ddagger}$ Institute of Applied Mathematics, AMSS, Beijing 100190, P.R. China (tengwang@amss.ac.cn). 
We take $A=R=\gamma-1$ in this paper for simplicity. Then the local sound speed is defined by

$$
c:=\sqrt{\gamma(\gamma-1) \theta}=\sqrt{\gamma(\gamma-1) \rho^{\gamma-1} e^{S}} .
$$

We are interested in the relation between the solutions of the compressible NavierStokes equations and those of the corresponding inviscid Euler equations:

$$
\left\{\begin{array}{l}
\rho_{t}+(\rho u)_{x}=0 \\
(\rho u)_{t}+\left(\rho u^{2}+p\right)_{x}=0 \\
{\left[\rho\left(e+\frac{u^{2}}{2}\right)\right]_{t}+\left[\rho u\left(e+\frac{u^{2}}{2}\right)+u p\right]_{x}=0 .}
\end{array}\right.
$$

The Euler system (1.6) is a strictly hyperbolic one for $\rho>0$ whose 1- and 3characteristic fields are genuinely nonlinear, that is, in the equivalent system

$$
\left(\begin{array}{c}
\rho \\
u \\
S
\end{array}\right)_{t}+\left(\begin{array}{ccc}
u & \rho & 0 \\
\gamma A \rho^{\gamma-2} e^{S} & u & A \rho^{\gamma-1} e^{S} \\
0 & 0 & u
\end{array}\right)\left(\begin{array}{l}
\rho \\
u \\
S
\end{array}\right)_{x}=0
$$

the Jacobi matrix

$$
\left(\begin{array}{ccc}
u & \rho & 0 \\
\gamma A \rho^{\gamma-2} e^{S} & u & A \rho^{\gamma-1} e^{S} \\
0 & 0 & u
\end{array}\right)
$$

has three distinct eigenvalues

$$
\lambda_{1}(\rho, u, S)=u-c, \quad \lambda_{2}(\rho, u, S)=u, \quad \lambda_{3}(\rho, u, S)=u+c,
$$

with corresponding right eigenvectors

$$
r_{1}(\rho, u, S)=(-\rho, c, 0)^{t}, \quad r_{3}(\rho, u, S)=(\rho, c, 0)^{t},
$$

such that

$$
r_{i}(\rho, u, S) \cdot \nabla_{(\rho, u, S)} \lambda_{i}(\rho, u, S)=\frac{\gamma+1}{2} c \neq 0, \quad i=1,3, \quad \forall \theta>0 .
$$

We can define two 1-Riemann invariants by

$$
\Sigma_{1}^{(1)}=u+\frac{2}{\gamma-1} c, \quad \Sigma_{1}^{(2)}=S,
$$

and two 3-Riemann invariants by

$$
\Sigma_{3}^{(1)}=u-\frac{2}{\gamma-1} c, \quad \Sigma_{3}^{(2)}=S,
$$

such that

$$
\nabla_{(\rho, u, S)} \Sigma_{i}^{(j)}(\rho, u, S) \cdot r_{i}(\rho, u, S) \equiv 0, \quad i=1,3, j=1,2 \quad \forall \rho>0 .
$$

The study of the limiting process of viscous flows, when the viscosity tends to zero, is one of the important problems in the theory of compressible fluids. From the 
kinetic theory, the viscosity $\epsilon$ and heat-conductivity coefficient $\kappa$ should be on the same order, so the following assumption is reasonable (see also [9]):

$$
\begin{cases}\kappa=O(\epsilon), & \text { as } \quad \epsilon \rightarrow 0 \\ \frac{\kappa(\epsilon)}{\epsilon} \geqq c>0, & \text { as } \quad \epsilon \rightarrow 0\end{cases}
$$

for some positive constant $c$. This indicates that the coefficient of heat conduction also tends to zero as the viscosity tends to zero, so we can take $\kappa=\epsilon$ in the following without loss of generality. The inviscid compressible flow contains singularities such as shock and the vacuum in general. Therefore, determining how to justify the zero dissipation limit of the Euler equations with basic wave patterns or the vacuum is a natural and difficult problem.

For the full Navier-Stokes equations which also have conservation of energy, there are also many results on the zero dissipation limit to the corresponding full Euler system with basic wave patterns without vacuum. We refer to Jiang-Ni-Sun [9] and Xin-Zeng [21] for the rarefaction wave, Wang [19] for the shock wave, Ma [14] for the contact discontinuity. Huang-Wang-Yang $[7,8]$ proved the vanishing viscosity of the compressible Euler equations for the cases of superposition of two rarefaction waves and a contact discontinuity and the superposition of one shock and one rarefaction wave. Recently, Huang-Wang-Wang-Yang [5] succeed in justifying the vanishing viscosity limit of compressible Navier-Stokes equations in the setting of Riemann solutions for the superposition of shock wave, rarefaction wave, and contact discontinuity. Jiu-Wang-Xin [10] studied the large time asymptotic behavior toward rarefaction wave for solutions to the one-dimensional isentropic compressible Navier-Stokes equations with density-dependent viscosity for general initial data whose far fields are connected by a rarefaction wave to the corresponding Euler equations with one end state being vacuum.

Now we give a description of the rarefaction wave connected to the vacuum to the compressible Euler equations (1.6); see also the reference [18]. For definiteness, 3 -rarefaction wave will be considered. If we investigate the compressible Euler system (1.6) with the Riemann initial data

$$
\begin{cases}\rho(0, x)=0, & x<0 \\ (\rho, u, \theta)(0, x)=\left(\rho_{+}, u_{+}, \theta_{+}\right) & , x>0\end{cases}
$$

where the left side is the vacuum state and $\rho_{+}>0, u_{+}, \theta_{+}>0$ are prescribed constants on the right state, then the Riemann problem (1.6), (1.9) admits a 3-rarefaction wave connected to the vacuum on the left side. By the fact that along the 3 -rarefaction wave curve, the 3 -Riemann invariant $\Sigma_{3}^{(i)}(\rho, u, \theta),(i=1,2)$ is constant in $(x, t)$, we can get the velocity $u_{-}=\Sigma_{3}^{(1)}\left(\rho_{+}, u_{+}, \theta_{+}\right)$being the speed of the fluid coming into the vacuum from the 3 -rarefaction wave. This 3 -rarefaction wave connecting the vacuum $\rho=0$ to $\left(\rho_{+}, u_{+}, \theta_{+}\right)$is the self-similar solution $\left(\rho^{r_{3}}, u^{r_{3}}, \theta^{r_{3}}\right)(\xi),\left(\xi=\frac{x}{t}\right)$ of (1.6) defined by

$$
\lambda_{3}\left(\rho^{r_{3}}(\xi), u^{r_{3}}(\xi), \theta^{r_{3}}(\xi)\right)= \begin{cases}\rho^{r_{3}}(\xi) \equiv 0, & \text { if } \xi<\lambda_{3}\left(0, u_{-}, 0\right)=u_{-}, \\ \xi, & \text { if } u_{-} \leq \xi \leq \lambda_{3}\left(\rho_{+}, u_{+}, \theta_{+}\right), \\ \lambda_{3}\left(\rho_{+}, u_{+}, \theta_{+}\right), & \text {if } \xi>\lambda_{3}\left(\rho_{+}, u_{+}, \theta_{+}\right),\end{cases}
$$

and

$$
\Sigma_{3}^{(1)}\left(\rho^{r_{3}}(\xi), u^{r_{3}}(\xi), \theta^{r_{3}}(\xi)\right)=\Sigma_{3}^{(1)}\left(0, u_{-}, 0\right)=\Sigma_{3}^{(1)}\left(\rho_{+}, u_{+}, \theta_{+}\right)
$$


Thus we can define the momentum $m^{r_{3}}$ and the total internal energy $\widetilde{e^{r_{3}}}$ of a 3 rarefaction wave by

$$
m^{r_{3}}(\xi):=\left\{\begin{array}{lll}
\rho^{r_{3}}(\xi) u^{r_{3}}(\xi), & \text { if } & \rho^{r_{3}}>0 \\
0, & \text { if } & \rho^{r_{3}}=0
\end{array}\right.
$$

and

$$
\widetilde{e^{r_{3}}}(\xi):=\left\{\begin{array}{lll}
\rho^{r_{3}}(\xi) \theta^{r_{3}}(\xi), & \text { if } \quad \rho^{r_{3}}>0 \\
0, & \text { if } & \rho^{r_{3}}=0
\end{array}\right.
$$

In the present paper, we want to construct a sequence of solutions $\left(\rho^{\epsilon}, m^{\epsilon}, \widetilde{e}^{\epsilon}:=\right.$ $\left.\rho^{\epsilon} \theta^{\epsilon}\right)(x, t)$ to the compressible Navier-Stokes equations (1.1) which converge to the 3 -rarefaction wave $\left(\rho^{r_{3}}, m^{r_{3}}, \widetilde{e^{r_{3}}}=\rho^{r_{3}} \theta^{r_{3}}\right)(x / t)$ defined above as $\epsilon$ tends to zero. The effects of initial layers will be ignored by choosing the well-prepared initial data depending on the viscosity for the Navier-Stokes equations.

The main novelty and difficulty of the paper is determining how to control the degeneracies caused by the vacuum in the rarefaction wave. To overcome this difficulty, we have to deal with the full compressible Navier-Stokes equations in Eulerian coordinates, rather than Lagrangian coordinates generally used in previous papers, because the Lagrangian transformation does not work when the vacuum state is involved in the fluid. However, the new convection term, which couples by density and velocity, complicates the calculations in the proof. Following our previous paper [3], we cut off the 3 -rarefaction wave with vacuum along the rarefaction wave curve. More precisely, for any $\mu>0$ to be determined, the cut-off rarefaction wave will connect the state $(\rho, u, \theta)=\left(\mu, u_{\mu}, e^{\bar{S}} \mu^{\gamma-1}\right)$ and $\left(\rho_{+}, u_{+}, \theta_{+}\right)$, where $u_{\mu}$ can be obtained uniquely by the definition of the 3-rarefaction wave curve; see Section 2 for more details. Then an approximate rarefaction wave to this cut-off rarefaction wave will be constructed through the Burgers equation. Finally, the desired solution sequences to the compressible Navier-Stokes equations (1.1) could be established around the approximate rarefaction wave. The new difficulty is that the temperature also degenerate in the vacuum region. In fact, we choose $\mu=\epsilon^{a}|\ln \epsilon|$ with $a$ defined in (1.15) to control the degeneracies caused by the vacuum in rarefaction wave. Compared with [3], we need a more delicate a priori assumptions (3.11) to carry out the energy estimates. With the balance the degeneracies between the density and the temperature, we can close the a priori assumptions and obtain the desired results.

Now we state our main result as follows.

THEOREM 1.1. Let $\left(\rho^{r_{3}}, m^{r_{3}}, \widetilde{e^{r_{3}}}\right)(x / t)$ be the 3-rarefaction wave defined by (1.10)(1.13) with one-side vacuum state. Then there exists a small positive constant $\epsilon_{0}$ such that for any $\epsilon \in\left(0, \epsilon_{0}\right)$, we can construct a family of global smooth solutions $\left(\rho^{\epsilon}, m^{\epsilon}=\right.$ $\left.\rho^{\epsilon} u^{\epsilon}, \widetilde{e}^{\epsilon}=\rho^{\epsilon} \theta^{\epsilon}\right)(x, t)$ to the compressible Navier-Stokes equations (1.1) satisfying

$$
\begin{array}{r}
\left(\rho^{\epsilon}-\rho^{r_{3}}, m^{\epsilon}-m^{r_{3}}, \widetilde{e^{\epsilon}}-\widetilde{e^{r_{3}}}\right),\left(\rho^{\epsilon}, m^{\epsilon}, \widetilde{e^{\epsilon}}\right)_{x} \in C^{0}\left(0,+\infty ; L^{2}(\mathbf{R})\right), \\
u_{x x}^{\epsilon}, \theta_{x x}^{\epsilon} \in L^{2}\left(0,+\infty ; L^{2}(\mathbf{R})\right) .
\end{array}
$$

(2) As viscosity $\epsilon \rightarrow 0,\left(\rho^{\epsilon}, m^{\epsilon}, \widetilde{e^{\epsilon}}\right)(x, t)$ converges to $\left(\rho^{r_{3}}, m^{r_{3}}, \widetilde{e^{r_{3}}}\right)(x / t)$ pointwise except at $(0,0)$. Furthermore, for any given positive constant $l$, there exists a constant 
$C_{l}>0$, independent of $\epsilon$, such that

$$
\begin{aligned}
& \sup _{t \geq l}\left\|\rho^{\epsilon}(\cdot, t)-\rho^{r_{3}}\left(\frac{\cdot}{\bar{t}}\right)\right\|_{L^{\infty}} \leq C_{l} \epsilon^{a}|\ln \epsilon|, \\
& \sup _{t \geq l}\left\|m^{\epsilon}(\cdot, t)-m^{r_{3}}(\cdot \dot{\bar{t}})\right\|_{L^{\infty}} \leq C_{l} \epsilon^{b}|\ln \epsilon|, \\
& \sup _{t \geq l}\left\|\widetilde{e^{\epsilon}}(\cdot, t)-\widetilde{e^{r_{3}}}\left(\cdot \frac{\cdot}{t}\right)\right\|_{L^{\infty}} \leq C_{l} \epsilon^{c}|\ln \epsilon|,
\end{aligned}
$$

with the positive constants $a, b$, and $c$ given by

$$
\begin{gathered}
a=\left\{\begin{array}{llc}
\frac{1}{2(\gamma+2)}, & \text { if } & 1<\gamma \leq 2, \\
\frac{1}{2(3 \gamma-2)}, & \text { if } & \gamma>2,
\end{array}\right. \\
b=\left\{\begin{array}{lll}
\frac{3}{8(\gamma+2)}, & \text { if } & 1<\gamma \leq 2, \\
\frac{4 \gamma-5}{8(3 \gamma-2)}, & \text { if } & 2<\gamma \leq \frac{9}{4}, \\
\frac{1}{2(3 \gamma-2)}, & \text { if } & \gamma>\frac{9}{4},
\end{array}\right.
\end{gathered}
$$

and

$$
c=\left\{\begin{array}{lll}
\frac{1}{8}, & \text { if } & 1<\gamma \leq 2 \\
\frac{1}{2(3 \gamma-2)}, & \text { if } & \gamma>2 .
\end{array}\right.
$$

REMARK 1.2. It is noted that in the a priori estimates (3.14) below, the estimates for $\phi^{2}$ and $\zeta^{2}$ from the potential energy hold with the weight $\bar{\rho}^{\gamma-2}$ and $\bar{\rho}^{2-\gamma}$ respectively which degenerate in the vacuum region. Therefore, the convergence rate obtained in Lemma 3.2 and thus in Theorem 1.1 depends on $\gamma$.

REMARK 1.3. It is also interesting to study the zero dissipation limit for the full compressible Navier-Stokes equations with variable viscosity. Li-Wang-Wang [11] generalized Theorem 1.1 to the full compressible Navier-Stokes equations (1.1) with temperature-dependent viscosity and heat-conduction coefficient.

The rest of the paper is organized as follows. In Section 2, we construct a smooth 3 -rarefaction wave which approximates the cut-off rarefaction wave based on the inviscid Burgers equation. The proof the Theorem 1.1 is given in Section 3.

Throughout this paper, $H^{k}(\mathbf{R}), k=0,1,2, \ldots$, denotes the $l$-th order Sobolev space with its norm

$$
\|f\|_{k}=\left(\sum_{j=0}^{k}\left\|\partial_{y}^{j} f\right\|^{2}\right)^{\frac{1}{2}}, \quad \text { and }\|\cdot\|:=\|\cdot\|_{L^{2}(d z)},
$$

while $L^{2}(d z)$ means the $L^{2}$ integral over $\mathbf{R}$ with respect to the Lebesgue measure $d z$, and $z=x$ or $y$. For simplicity, we also write $C$ as generic positive constants which are independent of time $t$ and viscosity and heat conductivity coefficient $\epsilon$ unless otherwise stated. 


\section{Rarefaction waves}

As in our previous paper [3], the approximate rarefaction wave to the compressible Navier-Stokes equations (1.1) can be constructed by the solution of Burger's equation

$$
\left\{\begin{array}{l}
w_{t}+w w_{x}=0 \\
w(0, x)=w_{\delta}(x)=w\left(\frac{x}{\delta}\right)=\frac{w_{+}+w_{-}}{2}+\frac{w_{+}-w_{-}}{2} \tanh \frac{x}{\delta},
\end{array}\right.
$$

where $\delta>0$ is a small parameter to be determined. In fact, we choose $\delta=\epsilon^{a}$ in (3.12) with $a$ given by (1.15) in the following. Note that the solution $w_{\delta}^{r}(t, x)$ of the problem (2.1) is given by

$$
w_{\delta}^{r}(t, x)=w_{\delta}\left(x_{0}(t, x)\right), \quad x=x_{0}(t, x)+w_{\delta}\left(x_{0}(t, x)\right) t .
$$

$w_{\delta}^{r}(t, x)$ has the following properties.

LEMma 2.1. ([20,3]) The problem (2.1) has a unique smooth global solution $w_{\delta}^{r}(x, t)$ for each $\delta>0$ such that

(1) $w_{-}<w_{\delta}^{r}(x, t)<w_{+}, \partial_{x} w_{\delta}^{r}(x, t)>0$, for $x \in \mathbf{R}, t \geq 0, \delta>0$.

(2) The following estimates hold for all $t>0, \delta>0$ and $p \in[1, \infty]$ :

$$
\begin{aligned}
\left\|\partial_{x} w_{\delta}^{r}(\cdot, t)\right\|_{L^{p}} & \leq C\left(w_{+}-w_{-}\right)^{1 / p}(\delta+t)^{-1+1 / p} \\
\left\|\partial_{x}^{2} w w^{r}(\dot{r}, t)\right\|_{L^{p}} & \leq C(\delta+t)^{-1} \delta^{-1+1 / p} \\
\left|\frac{\partial^{2} w_{\delta}^{r}(x, t)}{\partial x^{2}}\right| & \leq \frac{4}{\delta} \frac{\partial w_{\delta}^{r}(x, t)}{\partial x}
\end{aligned}
$$

(3) There exists a constant $\delta_{0} \in(0,1)$ such that for $\delta \in\left(0, \delta_{0}\right], t>0$,

$$
\left\|w_{\delta}^{r}(\cdot, t)-w^{r}\left(\frac{\cdot}{t}\right)\right\|_{L^{\infty}} \leq C \delta t^{-1}[\ln (1+t)+|\ln \delta|] .
$$

As mentioned in the introduction, we will cut off the 3-rarefaction wave with vacuum along the wave curve in order to overcome the degeneracies caused by the vacuum. More precisely, for any $\mu>0$ to be determined, we can get a state $(\rho, u, \theta)=\left(\mu, u_{\mu}, e^{\bar{S}} \mu^{\gamma-1}\right)$ belonging to the 3 -rarefaction wave curve, where $\bar{S}=S_{+}=$ $-(\gamma-1) \log \rho_{+}+\log \theta_{+}$. From the fact that the 3 -Riemann invariant $\Sigma_{3}^{(i)}(\rho, u, \theta),(i=$ $1,2)$ is constant along the 3-rarefaction wave curve, $u_{\mu}$ can be computed explicitly by $u_{\mu}=\Sigma_{3}^{(1)}\left(\rho_{+}, u_{+}, \theta_{+}\right)+2 \sqrt{\frac{\gamma}{\gamma-1} \mu^{\gamma-1} e^{S_{+}}}$. Now we get a new 3-rarefaction wave $\left(\rho_{\mu}^{r_{3}}, u_{\mu}^{r_{3}}, \theta_{\mu}^{r_{3}}\right)(\xi),(\xi=x / t)$ connecting the state $\left(\mu, u_{\mu}, e^{\bar{S}} \mu^{\gamma-1}\right)$ to the state $\left(\rho_{+}, u_{+}, \theta_{+}\right)$ which can be expressed explicitly by

$$
\lambda_{3}\left(\rho_{\mu}^{r_{3}}, u_{\mu}^{r_{3}}, \theta_{\mu}^{r_{3}}\right)(\xi)= \begin{cases}\lambda_{3}\left(\mu, u_{\mu}, e^{\bar{S}} \mu^{\gamma-1}\right), & \xi<\lambda_{3}\left(\mu, u_{\mu}, e^{\bar{S}} \mu^{\gamma-1}\right), \\ \xi, & \lambda_{3}\left(\mu, u_{\mu}, e^{\bar{S}} \mu^{\gamma-1}\right) \leq \xi \leq \lambda_{3}\left(\rho_{+}, u_{+}, \theta_{+}\right), \\ \lambda_{3}\left(\rho_{+}, u_{+}, \theta_{+}\right), & \xi>\lambda_{3}\left(\rho_{+}, u_{+}, \theta_{+}\right)\end{cases}
$$

and

$$
\Sigma_{3}^{(1)}\left(\rho_{\mu}^{r_{3}}, u_{\mu}^{r_{3}}, \theta_{\mu}^{r_{3}}\right)=\Sigma_{3}^{(1)}\left(\mu, u_{\mu}, e^{\bar{S}} \mu^{\gamma-1}\right)=\Sigma_{3}^{(1)}\left(\rho_{+}, u_{+}, \theta_{+}\right) .
$$

Correspondingly, we can define the momentum function and the total internal energy $m_{\mu}^{r_{3}}:=\rho_{\mu}^{r_{3}} u_{\mu}^{r_{3}}$ and $\widetilde{e_{\mu}^{r_{3}}}:=\rho_{\mu}^{r_{3}} \theta_{\mu}^{r_{3}}$ respectively. It is easy to show that the cut-off 3 rarefaction wave $\left(\rho_{\mu}^{r_{3}}, m_{\mu}^{r_{3}}, \widetilde{e_{\mu}^{r_{3}}}\right)(x / t)$ converges to the original 3-rarefaction wave with vacuum $\left(\rho^{r_{3}}, m^{r_{3}}, \widetilde{e^{r_{3}}}\right)(x / t)$ in the sup-norm with the convergence rate $\mu$ as $\mu$ tends to zero. More precisely, we have

Lemma 2.2. There exists a constant $\mu_{0} \in(0,1)$ such that for $\mu \in\left(0, \mu_{0}\right], t>0$, 


$$
\left\|\left(\rho_{\mu}^{r_{3}}, m_{\mu}^{r_{3}}, \widetilde{e_{\mu}^{r_{3}}}\right)(\cdot / t)-\left(\rho^{r_{3}}, m^{r_{3}}, \widetilde{e^{r_{3}}}\right)(\cdot / t)\right\|_{L^{\infty}} \leq C \mu .
$$

Now the approximate rarefaction wave $\left(\bar{\rho}_{\mu, \delta}, \bar{u}_{\mu, \delta}, \bar{\theta}_{\mu, \delta}\right)(x, t)$ of the cut-off 3rarefaction wave $\left(\rho_{\mu}^{r_{3}}, u_{\mu}^{r_{3}}, \theta_{\mu}^{r_{3}}\right)\left(\frac{x}{t}\right)$ to compressible Euler equations (1.6) can be defined by

$$
\left\{\begin{array}{l}
w_{+}=\lambda_{3}\left(\rho_{+}, u_{+}, \theta_{+}\right), \quad w_{-}=\lambda_{3}\left(\mu, u_{\mu}, e^{\bar{S}} \mu^{\gamma-1}\right), \\
w_{\delta}^{r}(t, x)=\lambda_{3}\left(\bar{\rho}_{\mu, \delta}, \bar{u}_{\mu, \delta}, \bar{\theta}_{\mu, \delta}\right)(t, x), \\
\Sigma_{3}^{(1)}\left(\bar{\rho}_{\mu, \delta}, \bar{u}_{\mu, \delta}, \bar{\theta}_{\mu, \delta}\right)(x, t)=\Sigma_{3}^{(1)}\left(\rho_{+}, u_{+}, \theta_{+}\right)=\Sigma_{3}^{(1)}\left(\mu, u_{\mu}, e^{\bar{S}} \mu^{\gamma-1}\right),
\end{array}\right.
$$

where $w_{\delta}^{r}$ is the solution of Burger's equation (2.1) defined in (2.2). From now on, the subscript of $\left(\bar{\rho}_{\delta, \mu}, \bar{u}_{\delta, \mu}, \bar{\theta}_{\delta, \mu}\right)(x, t)$ will be abbreviated as $(\bar{\rho}, \bar{u}, \bar{\theta})(x, t)$ for simplicity. Then the approximate cut-off 3 -rarefaction wave $(\bar{\rho}, \bar{u}, \bar{\theta})$ defined above satisfies

$$
\left\{\begin{array}{l}
\bar{\rho}_{t}+(\bar{\rho} \bar{u})_{x}=0, \\
(\bar{\rho} \bar{u})_{t}+\left(\bar{\rho} \bar{u}^{2}+R \bar{\rho} \bar{\theta}\right)_{x}=0, \\
{\left[\bar{\rho}\left(\bar{e}+\frac{\bar{u}^{2}}{2}\right)\right]_{t}+\left[\bar{\rho} \bar{u}\left(\bar{e}+\frac{\bar{u}^{2}}{2}\right)+\bar{u} \bar{p}\right]_{x}=0,}
\end{array}\right.
$$

and the properties of the approximate rarefaction wave $(\bar{\rho}, \bar{u}, \bar{\theta})$ are listed without proof in the following lemma.

Lemma 2.3. The approximate cut-off 3-rarefaction wave $(\bar{\rho}, \bar{u}, \bar{\theta})$ defined in (2.8) satisfies the following properties:

(i) $\bar{u}_{x}(x, t)=\frac{2}{\gamma+1}\left(w_{\delta}^{r}\right)_{x}>0$, for $x \in \mathbf{R}, t \geq 0$,

$$
\begin{aligned}
& \bar{\rho}_{x}=\frac{1}{\sqrt{\gamma(\gamma-1) e^{S_{+}}}} \bar{\rho}^{\frac{3-\gamma}{2}} \bar{u}_{x}, \\
& \bar{\rho}_{x x}=\frac{1}{\sqrt{\gamma(\gamma-1) e^{S_{+}}}} \bar{\rho}^{\frac{3-\gamma}{2}} \bar{u}_{x x}+\frac{3-\gamma}{2 \gamma(\gamma-1) e^{S_{+}}} \bar{\rho}^{2-\gamma}\left(\bar{u}_{x}\right)^{2}, \\
& \bar{\theta}_{x}=\sqrt{\frac{\gamma-1}{\gamma}} \bar{\theta}^{\frac{1}{2}} \bar{u}_{x}, \text { and } \bar{\theta}_{x x}=\sqrt{\frac{\gamma-1}{\gamma}} \bar{\theta}^{\frac{1}{2}} \bar{u}_{x x}+\frac{\gamma-1}{2 \gamma}\left(\bar{u}_{x}\right)^{2} .
\end{aligned}
$$

(ii) The following estimates hold for all $t>0, \delta>0$, and $p \in[1, \infty]$ :

$$
\begin{aligned}
& \left\|\bar{u}_{x}(\cdot, t)\right\|_{L^{p}} \leq C\left(w_{+}-w_{-}\right)^{1 / p}(\delta+t)^{-1+1 / p}, \\
& \left\|\bar{u}_{x x}(\cdot, t)\right\|_{L^{p}} \leq C(\delta+t)^{-1} \delta^{-1+1 / p}
\end{aligned}
$$

(iii) There exists a constant $\delta_{0} \in(0,1)$ such that for $\delta \in\left(0, \delta_{0}\right], t>0$,

$$
\left\|\left(\bar{\rho}-\rho_{\mu}^{r_{3}}, \bar{u}-u_{\mu}^{r_{3}}, \bar{\theta}-\theta_{\mu}^{r_{3}}\right)(\cdot, t)\right\|_{L^{\infty}} \leq C \delta t^{-1}[\ln (1+t)+|\ln \delta|] .
$$

\section{Proof of Theorem 1.1}

In order to prove Theorem 1.1, we regard the solution $\left(\rho^{\epsilon}, u^{\epsilon}, \theta^{\epsilon}\right)$ as the perturbation around the approximate rarefaction wave $(\bar{\rho}, \bar{u}, \bar{\theta})$. Consider the Cauchy problem for (1.1) with smooth initial data

$$
\left(\rho^{\epsilon}, u^{\epsilon}, \theta^{\epsilon}\right)(x, t=0)=(\bar{\rho}, \bar{u}, \bar{\theta})(x, 0) .
$$

Then we introduce the perturbation

$$
(\phi, \psi, \zeta)(y, \tau)=\left(\rho^{\epsilon}, u^{\epsilon}, \theta^{\epsilon}\right)(x, t)-(\bar{\rho}, \bar{u}, \bar{\theta})(x, t),
$$


where $y, \tau$ are the scaled variables

$$
y=\frac{x}{\epsilon}, \quad \tau=\frac{t}{\epsilon},
$$

and $\left(\rho^{\epsilon}, u^{\epsilon}, \theta^{\epsilon}\right)$ is assumed to be the solution to the problem (1.1). For the simplicity of the notation, we will omit the superscript of $\left(\rho^{\epsilon}, u^{\epsilon}, \theta^{\epsilon}\right)$ as $(\rho, u, \theta)$ from now on if there is no confusion of the notation. Substituting (3.2) and (3.3) into (1.1) and using the defining relation for $(\bar{\rho}, \bar{u}, \bar{\theta})$, we obtain

$$
\begin{aligned}
\phi_{\tau}+\rho \psi_{y}+u \phi_{y} & =-f, \\
\rho \psi_{\tau}+\rho u \psi_{y}+(\gamma-1)\left(\theta \phi_{y}+\rho \zeta_{y}\right)-\psi_{y y} & =-g, \\
\rho \zeta_{\tau}+\rho u \zeta_{y}-\zeta_{y y} & =-h, \\
(\phi, \psi, \zeta)(y, 0) & =0,
\end{aligned}
$$

where

$$
\left\{\begin{array}{l}
f=\bar{u}_{y} \phi+\bar{\rho}_{y} \psi \\
g=-\bar{u}_{y y}+\rho \psi \bar{u}_{y}+(\gamma-1)\left(\bar{\rho}_{y} \zeta-\frac{\bar{\rho}_{y} \bar{\theta} \phi}{\bar{\rho}}\right), \\
h=(\gamma-1)\left(\rho \theta \psi_{y}+\rho \zeta \bar{u}_{y}\right)+\rho \psi \bar{\theta}_{y}-\bar{\theta}_{y y}-\left(\bar{u}_{y}\right)^{2}-2 \bar{u}_{y} \psi_{y}-\left(\psi_{y}\right)^{2} .
\end{array}\right.
$$

We seek a global (in time) and bounded (in $L^{\infty}$ norm) solution $(\phi, \psi, \zeta)$ to the problem $(3.4)-(3.7)$. To this end, the solution space for $(3.4)-(3.7)$ is defined by

$$
\begin{gathered}
\chi\left(0, \tau_{1}(\epsilon)\right)=\left\{(\phi, \psi, \zeta) \mid(\phi, \psi, \zeta) \in C^{0}\left(\left[0, \tau_{1}(\epsilon)\right] ; H^{1}(\mathbf{R})\right), \quad \phi_{y} \in L^{2}\left(0, \tau_{1}(\epsilon) ; L^{2}(\mathbf{R})\right),\right. \\
\left.\psi_{y}, \zeta_{y} \in L^{2}\left(0, \tau_{1}(\epsilon) ; H^{1}(\mathbf{R})\right)\right\}
\end{gathered}
$$

with $0<\tau_{1}(\epsilon) \leq+\infty$.

THEOREM 3.1. There exist positive constants $\epsilon_{1}$ and $C$ independent of $\epsilon$, such that if $0<\epsilon<\epsilon_{1}$, then the problem (3.4) -(3.7) admits a unique global-in-time solution $(\phi, \psi, \zeta) \in \chi(0,+\infty)$ satisfying

$$
\begin{aligned}
& \sup _{\tau \in[0,+\infty)} \int_{\mathbf{R}}\left(\bar{\rho}^{\gamma-2} \phi^{2}+\bar{\rho} \psi^{2}+\bar{\rho}^{2-\gamma} \zeta^{2}+\frac{\phi_{y}^{2}}{\bar{\rho}^{3}}+\psi_{y}^{2}+\zeta_{y}^{2}\right) d y \\
& \quad+\int_{0}^{+\infty} \int_{\mathbf{R}}\left[\psi_{y}^{2}+\bar{\rho}^{1-\gamma} \zeta_{y}^{2}+\bar{\rho}^{\gamma-3} \phi_{y}^{2}+\left(\bar{\rho}^{\gamma-2} \phi^{2}+\bar{\rho} \psi^{2}+\bar{\rho}^{2-\gamma} \zeta^{2}+\psi_{y}^{2}+\zeta_{y}^{2}\right) \bar{u}_{y}\right. \\
& \left.+\frac{\zeta_{y y}^{2}}{\bar{\rho}}+\frac{\psi_{y y}^{2}}{\bar{\rho}}\right] d y d \tau \\
& \leq C \epsilon^{(1 / 2-\gamma a)}|\ln \epsilon|^{1 / 2-\gamma},
\end{aligned}
$$

where $a$ is given by (1.15). Consequently,

$$
\begin{aligned}
& \sup _{\tau \in[0,+\infty)}\|\phi(\cdot, \tau)\|_{L^{\infty}} \leq\left\{\begin{array}{lll}
C \epsilon^{\frac{1}{2(\gamma+2)}}|\ln \epsilon|^{1 / 4-\gamma / 2}, & \text { if } & 1<\gamma \leq 2, \\
C \epsilon^{\frac{1}{8}}|\ln \epsilon|^{3(1-\gamma) / 4}, & \text { if } & \gamma>2,
\end{array}\right. \\
& \sup _{\tau \in[0,+\infty)}\|\psi(\cdot, \tau)\|_{L^{\infty}} \leq\left\{\begin{array}{lll}
C \epsilon^{\frac{3}{8(\gamma+2)}}|\ln \epsilon|^{-\gamma / 2}, & \text { if } & 1<\gamma \leq 2, \\
C \epsilon^{\frac{4 \gamma-5}{8(3 \gamma-2)}}|\ln \epsilon|^{-\gamma / 2}, & \text { if } & \gamma>2,
\end{array}\right. \\
& \sup _{\tau \in[0,+\infty)}\|\zeta(\cdot, \tau)\|_{L^{\infty}} \leq\left\{\begin{array}{lll}
C \epsilon^{\frac{1}{8}}|\ln \epsilon|^{-(\gamma+1) / 4}, & \text { if } & 1<\gamma \leq 2, \\
C \epsilon^{\frac{\gamma-1}{2(3 \gamma-2)}}|\ln \epsilon|^{(1-2 \gamma) / 4}, & \text { if } & \gamma>2,
\end{array}\right.
\end{aligned}
$$


In what follows, the analysis is always carried out under the a priori assumptions

$$
\sup _{0 \leq \tau \leq \tau_{1}(\epsilon)}\|\phi(\cdot, \tau)\|_{L^{\infty}} \leq \epsilon^{a}, \sup _{0 \leq \tau \leq \tau_{1}(\epsilon)}\left\|\left(\psi_{y}, \zeta_{y}\right)(\cdot, \tau)\right\| \leq 1, \sup _{0 \leq \tau \leq \tau_{1}(\epsilon)}\|\zeta(\cdot, \tau)\|_{L^{\infty}} \leq \epsilon^{(\gamma-1) a}
$$

with $a$ given by (1.15), where $\left[0, \tau_{1}(\epsilon)\right]$ is the time interval in which the solution exists (which may depend on $\epsilon$ ). Take

$$
\mu=\epsilon^{a}|\ln \epsilon|, \quad \delta=\epsilon^{a}
$$

in what follows. Then $\mu \geq \bar{C} \epsilon^{a}$, where $\bar{C}=\max \left\{2,\left(2 e^{-\bar{S}}\right)^{\frac{1}{\gamma-1}}\right\}$ if $\epsilon \ll 1$. Under the a priori assumptions (3.11), we can get

$$
\frac{\bar{\rho}}{2} \leq \rho \leq \frac{3 \bar{\rho}}{2}, \quad \text { and } \quad \bar{\theta} \leq \theta \leq \frac{3 \bar{\theta}}{2} .
$$

In fact, if $\epsilon \ll 1$, then one has

$$
\rho=\bar{\rho}+\phi \geq \bar{\rho}-\|\phi\|_{L^{\infty}} \geq \bar{\rho}-\epsilon^{a} \geq \bar{\rho}-\frac{1}{2} \mu \geq \frac{\bar{\rho}}{2},
$$

and

$$
\rho=\bar{\rho}+\phi \leq \bar{\rho}+\|\phi\|_{L^{\infty}} \leq \bar{\rho}+\epsilon^{a} \leq \bar{\rho}+\frac{1}{2} \mu \leq \frac{3 \bar{\rho}}{2} .
$$

Similarly, note that $\bar{\theta}=\bar{\rho}^{\gamma-1} e^{\bar{S}} \geq \mu^{\gamma-1} e^{\bar{S}}$ by the definition of the rarefaction wave profile defined in (2.8), and it holds that

$$
\theta=\bar{\theta}+\zeta \geq \bar{\theta}-\|\zeta\|_{L^{\infty}} \geq \bar{\theta}-\epsilon^{a(\gamma-1)} \geq \bar{\theta}-\frac{e^{\bar{S}}}{2} \mu^{\gamma-1} \geq \bar{\theta}-\frac{\bar{\theta}}{2}=\frac{\bar{\theta}}{2}
$$

and

$$
\theta=\bar{\theta}+\zeta \leq \bar{\theta}+\|\zeta\|_{L^{\infty}} \leq \bar{\theta}+\epsilon^{a(\gamma-1)} \leq \bar{\theta}+\frac{e^{\bar{S}}}{2} \mu^{\gamma-1} \leq \bar{\theta}+\frac{\bar{\theta}}{2}=\frac{3 \bar{\theta}}{2} .
$$

Because the proof for the local existence of the solution to (3.4) - (3.7) is standard, we omit it for brevity. We use the continuity argument to prove Theorem 1.1 just like in our previous paper [3], and it is sufficient to show the following a priori estimates for fixed $\epsilon$.

Lemma 3.2. (A priori estimates) Let $\gamma>1$ and $(\phi, \psi, \zeta) \in \chi\left(0, \tau_{1}(\epsilon)\right)$ be a solution to the problem (3.4) - (3.7), where $\tau_{1}(\epsilon)$ is the maximum existence time of the solution satisfying the a priori assumptions (3.11). Then there exists positive constants $\epsilon_{2}$ such that if $0<\epsilon \leq \epsilon_{2}$, then

$$
\begin{gathered}
\sup _{0 \leq \tau \leq \tau_{1}(\epsilon)} \int_{\mathbf{R}}\left(\bar{\rho}^{\gamma-2} \phi^{2}+\bar{\rho} \psi^{2}+\bar{\rho}^{2-\gamma} \zeta^{2}+\frac{\phi_{y}^{2}}{\bar{\rho}^{3}}+\psi_{y}^{2}+\zeta_{y}^{2}\right) d y \\
+\int_{0}^{\tau_{1}(\epsilon)} \int_{\mathbf{R}}\left[\psi_{y}^{2}+\bar{\rho}^{1-\gamma} \zeta_{y}^{2}+\bar{\rho}^{\gamma-3} \phi_{y}^{2}+\left(\bar{\rho}^{\gamma-2} \phi^{2}+\bar{\rho} \psi^{2}+\bar{\rho}^{2-\gamma} \zeta^{2}+\psi_{y}^{2}+\zeta_{y}^{2}\right) \bar{u}_{y}\right. \\
\left.+\frac{\zeta_{y y}^{2}}{\bar{\rho}}+\frac{\psi_{y y}^{2}}{\bar{\rho}}\right] d y d \tau
\end{gathered}
$$




$$
\leq C \epsilon^{(1 / 2-\gamma a)}|\ln \epsilon|^{1 / 2-\gamma} .
$$

Consequently,

$$
\begin{aligned}
\sup _{0 \leq \tau \leq \tau_{1}(\epsilon)}\|\phi(\cdot, \tau)\|_{L^{\infty}} \leq \begin{cases}C \epsilon^{\frac{1}{2(\gamma+2)}|\ln \epsilon|^{1 / 4-\gamma / 2},} & \text { if } 1<\gamma \leq 2, \\
C \epsilon^{\frac{1}{8}}|\ln \epsilon|^{3(1-\gamma) / 4}, & \text { if } \quad \gamma>2,\end{cases} \\
\sup _{0 \leq \tau \leq \tau_{1}(\epsilon)}\|\psi(\cdot, \tau)\|_{L^{\infty}} \leq\left\{\begin{array}{lll}
C \epsilon^{\frac{3}{8(\gamma+2)}}|\ln \epsilon|^{-\gamma / 2}, & \text { if } & 1<\gamma \leq 2, \\
C \epsilon^{\frac{4 \gamma-5}{8(3 \gamma-2)}}|\ln \epsilon|^{-\gamma / 2}, & \text { if } & \gamma>2,
\end{array}\right. \\
\sup _{0 \leq \tau \leq \tau_{1}(\epsilon)}\|\zeta(\cdot, \tau)\|_{L^{\infty}} \leq\left\{\begin{array}{lll}
C \epsilon^{\frac{1}{8}}|\ln \epsilon|^{-(\gamma+1) / 4}, & \text { if } 1<\gamma \leq 2, \\
C \epsilon^{\frac{\gamma-1}{2(3 \gamma-2)}}|\ln \epsilon|^{(1-2 \gamma) / 4}, & \text { if } \quad \gamma>2,
\end{array}\right.
\end{aligned}
$$

where $a$ is given by (1.15) and the constant $C$ is independent of $\epsilon$ and $\tau_{1}(\epsilon)$.

Proof. (Proof of Lemma 3.2) The proof of Lemma 3.2 consists of the following steps.

Step 1. First, inspired by [13], we define the relative entropy-entropy flux pair $(\eta, q)$ as

$$
\left\{\begin{array}{l}
\eta=-\bar{\theta}\left\{\rho S-\bar{\rho} \bar{S}-\left.\nabla_{\mathbf{X}}(\rho S)\right|_{\mathbf{X}=\overline{\mathbf{X}}} \cdot(\mathbf{X}-\overline{\mathbf{X}})\right\} \\
q=-\bar{\theta}\left\{\rho u S-\bar{\rho} \bar{u} \bar{S}-\left.\nabla_{\mathbf{X}}(\rho S)\right|_{\mathbf{X}=\overline{\mathbf{X}}} \cdot(\mathbf{Y}-\overline{\mathbf{Y}})\right\}
\end{array}\right.
$$

where

$$
\begin{aligned}
& \mathbf{X}=\left(\rho, \rho u, \rho\left(\theta+\frac{u^{2}}{2}\right)\right), \\
& \mathbf{Y}=\left(\rho u, \rho u^{2}+(\gamma-1) \rho \theta, \rho u\left(\gamma \theta+\frac{u^{2}}{2}\right)\right) .
\end{aligned}
$$

A simple computation implies

$$
\begin{aligned}
& (\rho S)_{\rho}=S+\frac{u^{2}}{2 \theta}-\gamma, \\
& (\rho S)_{m}=-\frac{u^{2}}{\theta}, \\
& (\rho S)_{E}=\frac{1}{\theta}
\end{aligned}
$$

where $m=\rho u$ and $E=\rho\left(\theta+\frac{u^{2}}{2}\right)$, and then we get

$$
\left\{\begin{aligned}
\eta & =\rho \theta-\bar{\theta} \rho S+\rho\left[(\bar{S}-\gamma) \bar{\theta}+\frac{1}{2}|u-\bar{u}|^{2}\right]+(\gamma-1) \bar{\rho} \bar{\theta} \\
& =(\gamma-1) \rho \bar{\theta} \Phi\left(\frac{\bar{\rho}}{\rho}\right)+\frac{\rho \psi^{2}}{2}+\rho \bar{\theta} \Phi\left(\frac{\theta}{\theta}\right) \\
q & =u \eta+(\gamma-1)(u-\bar{u})(\rho \theta-\bar{\rho} \bar{\theta})
\end{aligned}\right.
$$

with

$$
\Phi(\eta):=\eta-\ln \eta-1
$$

Direct computations yield

$$
\begin{aligned}
& \eta_{\tau}+\left[q-\psi_{y} \psi-\frac{\zeta \zeta_{y}}{\theta}\right]_{y}+H \bar{u}_{y}+\left(\frac{\bar{\theta}}{\theta} \psi_{y}^{2}+\frac{\bar{\theta}}{\theta^{2}} \zeta_{y}^{2}\right) \\
= & \frac{\zeta}{\theta} \bar{\theta}_{y y}+\bar{u}_{y y} \psi+\frac{\bar{\theta}_{y} \zeta \zeta_{y}}{\theta^{2}}+\frac{\zeta}{\theta}\left(\bar{u}_{y}^{2}+2 \bar{u}_{y} \psi_{y}\right),
\end{aligned}
$$


where

$$
\begin{aligned}
H= & \rho(u-\bar{u})^{2}+(\gamma-1) \rho \bar{\theta} \Phi\left(\frac{\theta}{\bar{\theta}}\right)+(\gamma-1)^{2} \rho \bar{\theta} \Phi\left(\frac{\bar{\rho}}{\rho}\right) \\
& +\sqrt{\frac{\gamma-1}{\gamma}} \bar{\theta}^{\frac{1}{2}} \rho(u-\bar{u})\left((\gamma-1) \log \frac{\bar{\rho}}{\rho}+\log \frac{\theta}{\bar{\theta}}\right) \\
\geq & (1-\varepsilon) \rho(u-\bar{u})^{2}+(\gamma-1) \rho \bar{\theta}\left[\Phi\left(\frac{\theta}{\bar{\theta}}\right)+(\gamma-1) \Phi\left(\frac{\bar{\rho}}{\rho}\right)-\frac{1}{4 \varepsilon \gamma}\left((\gamma-1) \log \frac{\bar{\rho}}{\rho}+\log \frac{\theta}{\bar{\theta}}\right)^{2}\right]
\end{aligned}
$$

with $\varepsilon>0$ to be determined later. We say that

$$
H \geq C\left[\bar{\rho} \psi^{2}+\frac{\bar{\theta}}{\bar{\rho}} \phi^{2}+\frac{\bar{\rho}}{\bar{\theta}} \zeta^{2}\right]
$$

In fact, let

$$
x=\frac{\theta}{\bar{\theta}}, \quad y=\frac{\bar{\rho}}{\rho} .
$$

Under a priori assumptions (3.11), one has $x, y \sim 1$ as $\epsilon \rightarrow 0$. Considering the function

$$
f(x, y)=x-\log x-1+(\gamma-1)(y-\log y-1)-\frac{1}{4 \varepsilon \gamma}((\gamma-1) \log y+\log x)^{2},
$$

it follows that

$$
\begin{aligned}
& f_{x x}(x, y)=\frac{1}{x^{2}}-\frac{1}{2 \varepsilon \gamma x^{2}}[1-((\gamma-1) \log y+\log x)], \\
& f_{x y}(x, y)=\frac{1-\gamma}{2 \varepsilon \gamma} \frac{1}{x y} \\
& f_{y y}(x, y)=(\gamma-1) \frac{1}{y^{2}}\left\{1-\frac{1}{2 \varepsilon \gamma}[(\gamma-1)-((\gamma-1) \log y+\log x)]\right\} .
\end{aligned}
$$

It is easy to check that

$$
f(1,1)=f_{x}(1,1)=f_{y}(1,1)=0,
$$

and the Hessian matrix of $f$ at point $(1,1)$ is

$$
\nabla^{2} f(1,1)=\left(\begin{array}{cc}
1-\frac{1}{2 \varepsilon \gamma} & \frac{1-\gamma}{2 \varepsilon \gamma} \\
\frac{1-\gamma}{2 \varepsilon \gamma} & (\gamma-1)\left(1-\frac{\gamma-1}{2 \varepsilon \gamma}\right)
\end{array}\right),
$$

and is positive near the point $(1,1)$ if we set $\varepsilon=\frac{3}{4}$. Then $(3.23)$ is obtained under a priori assumptions (3.11).

Then integrating the equation (3.21) over $\mathbf{R}^{1} \times[0, \tau]$ and using (3.13) imply

$$
\begin{aligned}
& \int_{\mathbf{R}}\left(\bar{\rho}^{\gamma-2} \phi^{2}+\bar{\rho} \psi^{2}+\bar{\rho}^{2-\gamma} \zeta^{2}\right)(\tau, y) d y \\
& +\int_{0}^{\tau} \int_{\mathbf{R}}\left[\bar{u}_{y}\left(\bar{\rho}^{\gamma-2} \phi^{2}+\bar{\rho} \psi^{2}+\bar{\rho}^{2-\gamma} \zeta^{2}\right)+\psi_{y}^{2}+\frac{\zeta_{y}^{2}}{\bar{\theta}}\right] d y d \tau
\end{aligned}
$$




$$
\begin{aligned}
& \leq C \int_{0}^{\tau} \int_{\mathbf{R}}\left|\frac{\zeta}{\theta} \bar{\theta}_{y y}+\bar{u}_{y y} \psi+\frac{\zeta}{\theta} \bar{u}_{y}^{2}+\frac{\zeta}{\theta}\left(2 \psi_{y} \bar{u}_{y}+\frac{\bar{\theta}_{y} \zeta_{y}}{\theta}\right)\right| d y d \tau \\
& :=\sum_{i=1}^{4} I_{i} .
\end{aligned}
$$

By Sobolev's inequality and Lemma 2.3, we can obtain

$$
\begin{aligned}
& I_{1}= \int_{0}^{\tau} \int_{\mathbf{R}}\left|\bar{\theta}_{y y} \frac{\zeta}{\theta}\right| d y d \tau \\
& \leq C \mu^{-(\gamma-1) / 2} \int_{0}^{\tau}\left\|\bar{\theta}_{y y}\right\|_{L^{1}}\left\|\frac{\zeta}{\bar{\theta}^{1 / 2}}\right\|^{1 / 2}\left(\left\|\frac{\zeta_{y}}{\bar{\theta}^{1 / 2}}\right\|^{1 / 2}+\left\|\frac{\bar{\theta}_{y} \zeta}{\bar{\theta}^{3 / 2}}\right\|^{1 / 2}\right) d \tau \\
& \leq C \mu^{-(\gamma-1) / 2} \int_{0}^{\tau} \frac{1}{\tau+\delta / \epsilon}\left\|\frac{\zeta}{\bar{\theta}^{1 / 2}}\right\|^{1 / 2}\left\|\frac{\zeta_{y}}{\bar{\theta}^{1 / 2}}\right\|^{1 / 2} d \tau \\
& \quad+C \mu^{-(\gamma-1) / 2}\left(\frac{\epsilon}{\mu^{\gamma} \delta}\right)^{1 / 4} \int_{0}^{\tau} \frac{1}{\tau+\delta / \epsilon}\left\|\frac{\zeta}{\bar{\theta}^{1 / 2}}\right\|^{1 / 2} \| \sqrt{\bar{u}_{y} \bar{\rho}^{2-\gamma} \zeta \|^{1 / 2} d \tau} \\
& \leq \frac{1}{8} \int_{0}^{\tau}\left\|\frac{\zeta_{y}}{\bar{\theta}^{1 / 2}}\right\|^{2} d \tau+\frac{1}{8} \int_{0}^{\tau}\left\|\sqrt{\bar{u}_{y} \bar{\rho}^{2-\gamma} \zeta \|^{2}} d \tau+C \mu^{-\frac{2(\gamma-1)}{3}} \int_{0}^{\tau}\left(\frac{1}{\tau+\delta / \epsilon}\right)^{\frac{4}{3}}\right\| \frac{\zeta}{\bar{\theta}^{1 / 2}} \|^{\frac{2}{3}} d \tau \\
& \leq \frac{1}{8} \int_{0}^{\tau}\left\|\frac{\zeta_{y}}{\bar{\theta}^{1 / 2}}\right\|^{2} d \tau+\frac{1}{8} \int_{0}^{\tau}\left\|\sqrt{\bar{u}_{y} \bar{\rho}^{2-\gamma} \zeta \|^{2}} d \tau+\frac{1}{8} \sup _{\left[0, \tau_{1}(\epsilon)\right]}\right\| \bar{\rho}^{(2-\gamma) / 2} \zeta \|^{2} \\
& \quad+C\left(\mu^{(1-2 \gamma) / 3} \int_{0}^{\infty}\left(\frac{1}{\tau+\delta / \epsilon}\right)^{4 / 3} d \tau\right)^{3 / 2} \\
& \leq \frac{1}{8} \int_{0}^{\tau}\left\|\frac{\zeta_{y}}{\bar{\theta}^{1 / 2}}\right\|^{2} d \tau+\frac{1}{8} \int_{0}^{\tau}\left\|\sqrt{\bar{u}_{y} \bar{\rho}^{2-\gamma}} \zeta\right\|^{2} d \tau+\frac{1}{8} \sup _{\left[0, \tau_{1}(\epsilon)\right]}\left\|\bar{\rho}^{(2-\gamma) / 2} \zeta\right\|^{2} \\
& \quad+C \mu^{1 / 2-\gamma}\left(\frac{\epsilon}{\delta}\right)^{1 / 2},
\end{aligned}
$$

where we have used the fact that

$$
C \mu^{-\gamma} \delta^{-1} \epsilon=C \epsilon^{1-a-a \gamma}|\ln \epsilon|^{-\gamma} \leq C|\ln \epsilon|^{-\gamma} \leq \frac{1}{8}, \quad \text { if } \epsilon \ll 1 .
$$

By Sobolev's inequality and Cauchy's inequality, we can get

$$
\begin{aligned}
I_{2} & =\int_{0}^{\tau} \int_{\mathbf{R}} \psi \bar{u}_{y y} d y d \tau \\
& \leq C \int_{0}^{\tau}\left\|\bar{u}_{y y}\right\|_{L^{1}}\|\psi\|^{1 / 2}\left\|\psi_{y}\right\|^{1 / 2} d \tau \leq C \int_{0}^{\tau} \frac{1}{\tau+\delta / \epsilon}\|\psi\|\left\|^{1 / 2}\right\| \psi_{y} \|^{1 / 2} d \tau \\
& \leq \frac{1}{8} \int_{0}^{\tau} \int_{\mathbf{R}} \psi_{y}^{2} d y d \tau+C \int_{0}^{\tau}\left(\frac{1}{\tau+\epsilon / \delta}\right)^{4 / 3}\|\psi\|^{2 / 3} d \tau \\
& \leq \frac{1}{8} \int_{0}^{\tau} \int_{\mathbf{R}} \psi_{y}^{2} d y d \tau+C \mu^{-\frac{1}{3}} \sup _{\left[0, \tau_{1}(\epsilon)\right]}\|\sqrt{\bar{\rho}} \psi\|^{\frac{2}{3}} \int_{0}^{\tau}\left(\frac{1}{\tau+\delta / \epsilon}\right)^{4 / 3} d \tau \\
& \leq \frac{1}{8} \int_{0}^{\tau} \int_{\mathbf{R}} \psi_{y}^{2} d y d \tau+\frac{1}{8} \int_{0}^{\tau} \sup _{\left[0, \tau_{1}(\epsilon)\right]}\|\sqrt{\bar{\rho}} \psi\|^{2} d \tau+C\left(\frac{\epsilon}{\mu \delta}\right)^{\frac{1}{2}} \\
& \leq \frac{1}{8} \int_{0}^{\tau} \int_{\mathbf{R}} \psi_{y}^{2} d y d \tau+\frac{1}{8} \int_{0}^{\tau} \sup _{\left[0, \tau_{1}(\epsilon)\right]}\|\sqrt{\bar{\rho}} \psi\|^{2} d \tau+C\left(\frac{\epsilon}{\mu^{2 \gamma-1} \delta}\right)^{\frac{1}{2}} .
\end{aligned}
$$


Recalling Lemma 2.3, and Cauchy's inequality, it holds that

$$
\begin{aligned}
I_{3} & =\int_{0}^{\tau} \int_{\mathbf{R}} \frac{\zeta}{\theta} \bar{u}_{y}^{2} d y d \tau \\
& \leq \frac{1}{8} \int_{0}^{\tau} \int_{\mathbf{R}} \bar{u}_{y} \bar{\rho}^{2-\gamma} \zeta^{2} d y d \tau+C \int_{0}^{\tau} \int_{\mathbf{R}} \bar{\rho}^{\gamma-2} \bar{\theta}^{-2} \bar{u}_{y}^{3} d y d \tau \\
& \leq \frac{1}{8} \int_{0}^{\tau} \int_{\mathbf{R}} \bar{u}_{y} \bar{\rho}^{2-\gamma} \zeta^{2} d y d \tau+C \mu^{-\gamma} \int_{0}^{\tau}\left\|\bar{u}_{y}\right\|_{L^{3}}^{3} d \tau \\
& \leq \frac{1}{8} \int_{0}^{\tau} \int_{\mathbf{R}} \bar{u}_{y} \bar{\rho}^{2-\gamma} \zeta^{2} d y d \tau+C \mu^{-\gamma} \int_{0}^{\tau}\left(\frac{1}{\tau+\delta / \epsilon}\right)^{2} d \tau \\
& \leq \frac{1}{8} \int_{0}^{\tau} \int_{\mathbf{R}} \bar{u}_{y} \bar{\rho}^{2-\gamma} \zeta^{2} d y d \tau+C \frac{\epsilon}{\mu^{\gamma} \delta} \\
& \leq \frac{1}{8} \int_{0}^{\tau} \int_{\mathbf{R}} \bar{u}_{y} \bar{\rho}^{2-\gamma} \zeta^{2} d y d \tau+C \epsilon^{\frac{1}{2}-\gamma a}|\ln \epsilon|^{\frac{1}{2}-\gamma},
\end{aligned}
$$

where we have used the fact that

$$
C \frac{\epsilon}{\mu^{\gamma} \delta}=C \epsilon^{1-a-a \gamma}|\ln \epsilon|^{-\gamma} \leq C \epsilon^{\frac{1}{2}-\gamma a}|\ln \epsilon|^{\frac{1}{2}-\gamma}, \quad \text { if } \epsilon \ll 1 .
$$

Similarly,

$$
\begin{aligned}
I_{4} & =\int_{0}^{\tau} \int_{\mathbf{R}} \frac{\zeta}{\theta}\left(2 \bar{u}_{y} \psi_{y}+\frac{\bar{\theta}_{y} \zeta_{y}}{\theta}\right) d y d \tau \\
& \leq C \int_{0}^{\tau} \int_{\mathbf{R}} \bar{\rho}^{-\frac{\gamma}{2}} \bar{u}_{y}\left|\sqrt{\bar{\rho}^{2-\gamma}} \zeta\right|\left(\left|\psi_{y}\right|+\bar{\theta}^{-\frac{1}{2}}\left|\zeta_{y}\right|\right) d y d \tau \\
& \leq \frac{1}{8} \int_{0}^{\tau} \int_{\mathbf{R}}\left(\psi_{y}^{2}+\frac{\zeta_{y}^{2}}{\bar{\theta}}\right) d y d \tau+C \int_{0}^{\tau} \int_{\mathbf{R}} \bar{\rho}^{2-2 \gamma} \bar{u}_{y}^{2} \zeta^{2} d y d \tau \\
& \leq \frac{1}{8} \int_{0}^{\tau} \int_{\mathbf{R}}\left(\psi_{y}^{2}+\frac{\zeta_{y}^{2}}{\bar{\theta}}\right) d y d \tau+C \frac{\epsilon}{\mu^{\gamma} \delta} \int_{0}^{\tau} \int_{\mathbf{R}} \bar{u}_{y} \bar{\rho}^{2-\gamma} \zeta^{2} d y d \tau \\
& \leq \frac{1}{8} \int_{0}^{\tau} \int_{\mathbf{R}}\left(\psi_{y}^{2}+\frac{\zeta_{y}^{2}}{\bar{\theta}}\right) d y d \tau+\frac{1}{8} \int_{0}^{\tau} \int_{\mathbf{R}} \bar{u}_{y} \bar{\rho}^{2-\gamma} \zeta^{2} d y d \tau .
\end{aligned}
$$

Combining (3.24)-(3.28) and recalling (3.12) yields

$$
\begin{aligned}
& \int_{\mathbf{R}}\left(\bar{\rho}^{\gamma-2} \phi^{2}+\bar{\rho} \psi^{2}+\bar{\rho}^{2-\gamma} \zeta^{2}\right) d y \\
& +\int_{0}^{\tau} \int_{\mathbf{R}}\left[\psi_{y}^{2}+\bar{\rho}^{1-\gamma} \zeta_{y}^{2}+\left(\bar{\rho}^{\gamma-2} \phi^{2}+\bar{\rho} \psi^{2}+\bar{\rho}^{2-\gamma} \zeta^{2}\right) \bar{u}_{y}\right] d y d \tau \\
\leq & C \epsilon^{(1 / 2-\gamma a)}|\ln \epsilon|^{1 / 2-\gamma} .
\end{aligned}
$$

Step 2. We make use of the idea in [4] with modifications to derive the estimation of $\phi_{y}$. Differentiating (3.4) with respect to $y$ and then multiplying the resulted equation by $\phi_{y} / \rho^{3}$ yields

$$
\left(\frac{\phi_{y}^{2}}{2 \rho^{3}}\right)_{\tau}+\left(\frac{u \phi_{y}^{2}}{2 \rho^{3}}\right)_{y}+\frac{\psi_{y y} \phi_{y}}{\rho^{2}}=-\frac{\phi_{y}}{\rho^{3}}\left(\bar{u}_{y y} \phi+\bar{\rho}_{y y} \psi+2 \bar{\rho}_{y} \psi_{y}\right) .
$$


Multiplying (3.5) by $\phi_{y} / \rho^{2}$ gives

$$
\begin{aligned}
\left(\frac{\psi \phi_{y}}{\rho}\right)_{\tau}-\left(\frac{\psi \phi_{\tau}}{\rho}+\right. & \left.\bar{\rho}_{y} \frac{\psi^{2}}{\rho}\right)_{y}-\psi_{y}^{2}+(\gamma-1) \frac{\theta \phi_{y}^{2}}{\rho^{2}}+(\gamma-1) \frac{\zeta_{y} \phi_{y}}{\rho}-\frac{\psi_{y y} \phi_{y}}{\rho^{2}} \\
& -\bar{u}_{y} \frac{\psi_{y} \phi}{\rho}+2 \bar{\rho}_{y} \frac{\psi \psi_{y}}{\rho}+\bar{\rho}_{y y} \frac{\psi^{2}}{\rho}+\bar{\rho}_{y} \bar{u}_{y} \frac{\psi \phi}{\rho^{2}}-\bar{\rho} \bar{u}_{y} \frac{\psi \phi_{y}}{\rho^{2}}=-g \frac{\phi_{y}}{\rho^{2}} .
\end{aligned}
$$

Adding (3.30) and (3.31) together, then integrating the resulting equation over $\mathbf{R}^{1} \times$ $[0, \tau]$ implies

$$
\begin{gathered}
\int_{\mathbf{R}}\left(\frac{\phi_{y}^{2}}{2 \rho^{3}}+\frac{\psi \phi_{y}}{\rho}\right) d y+\int_{0}^{\tau} \int_{\mathbf{R}}(\gamma-1) \frac{\phi_{y}^{2}}{\rho^{3-\gamma}}+(\gamma-1) \frac{\zeta_{y} \phi_{y}}{\rho} d y d \tau \\
=\int_{0}^{\tau} \int_{\mathbf{R}}\left\{\psi_{y}^{2}+\bar{u}_{y} \frac{\psi_{y} \phi}{\rho}-2 \bar{\rho}_{y} \frac{\psi \psi_{y}}{\rho}-\bar{\rho}_{y y} \frac{\psi^{2}}{\rho}-\bar{\rho}_{y} \bar{u}_{y} \frac{\psi \phi}{\rho^{2}}+\bar{\rho} \bar{u}_{y} \frac{\psi \phi_{y}}{\rho^{2}}\right. \\
\left.-\frac{\phi_{y}}{\rho^{3}}\left(\bar{u}_{y y} \phi+\bar{\rho}_{y y} \psi+2 \bar{\rho}_{y} \psi_{y}\right)-g \frac{\phi_{y}}{\rho^{2}}\right\} d y d \tau .
\end{gathered}
$$

Combining (3.29) and (3.32) leads to

$$
\begin{aligned}
& \int_{\mathbf{R}}\left(\frac{\phi_{y}^{2}}{\bar{\rho}^{3}}+\bar{\rho} \psi^{2}+\bar{\rho}^{\gamma-2} \phi^{2}+\bar{\rho}^{2-\gamma} \zeta^{2}\right) d y \\
& +\int_{0}^{\tau} \int_{\mathbf{R}}\left(\psi_{y}^{2}+\bar{\rho}^{1-\gamma} \zeta_{y}^{2}+\bar{\rho}^{\gamma-3} \phi_{y}^{2}+\left(\bar{\rho}^{\gamma-2} \phi^{2}+\bar{\rho} \psi^{2}+\bar{\rho}^{2-\gamma} \zeta^{2}\right) \bar{u}_{y}\right) d y d \tau \\
\leq & C \int_{0}^{\tau} \int_{\mathbf{R}}\left\{\left|\bar{u}_{y} \frac{\psi_{y} \phi}{\bar{\rho}}\right|+\left|\bar{u}_{y} \frac{\psi \phi_{y}}{\bar{\rho}}\right|+\left|\bar{\rho}_{y} \frac{\psi \psi_{y}}{\bar{\rho}}\right|+\left|\bar{\rho}_{y} \frac{\phi_{y} \psi_{y}}{\bar{\rho}^{3}}\right|\right\} d y d \tau \\
& +C \int_{0}^{\tau} \int_{\mathbf{R}}\left\{\left|\bar{\rho}_{y y} \frac{\psi^{2}}{\bar{\rho}}\right|+\left|\bar{\rho}_{y y} \frac{\phi_{y} \psi}{\bar{\rho}^{3}}\right|+\left|\bar{u}_{y y} \frac{\phi_{y} \phi}{\bar{\rho}^{3}}\right|+\left|\bar{\rho}_{y} \bar{u}_{y} \frac{\psi \phi}{\bar{\rho}^{2}}\right|\right\} d y d \tau \\
& +C \int_{0}^{\tau} \int_{\mathbf{R}}\left|g \frac{\phi_{y}}{\bar{\rho}^{2}}\right| d y d \tau+C \epsilon^{(1 / 2-\gamma a)}|\ln \epsilon|^{1 / 2-\gamma} \\
:= & \sum_{i=1}^{3} J_{i}+C \epsilon^{(1 / 2-\gamma a)}|\ln \epsilon|^{1 / 2-\gamma} .
\end{aligned}
$$

Now we estimate the terms on the right-hand side of (3.33) one by one. By Lemma 2.3 , (3.13), and Cauchy's inequality, it holds that

$$
\begin{aligned}
J_{1} \leq & C \int_{0}^{\tau} \int_{\mathbf{R}} \bar{\rho}^{-\frac{\gamma}{2}} \bar{u}_{y}\left(\left|\psi_{y}\right|\left|\sqrt{\bar{\rho}^{\gamma-2}} \phi\right|+\left|\sqrt{\bar{\rho}^{\gamma-3}} \phi_{y}\right||\sqrt{\bar{\rho}} \psi|+\left|\psi_{y}\right||\sqrt{\bar{\rho}} \psi|\right) d y d \tau \\
& \quad+C \int_{0}^{\tau} \int_{\mathbf{R}} \bar{\rho}^{-\gamma} \bar{u}_{y}\left|\sqrt{\bar{\rho}^{\gamma-3}} \phi_{y}\right|\left|\psi_{y}\right| d y d \tau \\
\leq & \left(\frac{1}{16}+C \frac{\epsilon}{\mu^{\gamma} \delta}\right) \int_{0}^{\tau} \int_{\mathbf{R}}\left(\bar{\rho}^{\gamma-3} \phi_{y}^{2}+\psi_{y}^{2}\right) d y d \tau+C \frac{\epsilon}{\mu^{\gamma} \delta} \int_{0}^{\tau} \int_{\mathbf{R}} \bar{u}_{y}\left(\bar{\rho}^{\gamma-2} \phi^{2}+\bar{\rho} \psi^{2}\right) d y d \tau \\
\leq & \frac{1}{8} \int_{0}^{\tau} \int_{\mathbf{R}} \bar{\rho}^{\gamma-3} \phi_{y}^{2} d y d \tau+C \epsilon^{(1 / 2-\gamma a)}|\ln \epsilon|^{1 / 2-\gamma}
\end{aligned}
$$

Similarly,

$$
J_{2} \leq C \int_{0}^{\tau} \int_{\mathbf{R}}\left(\frac{\epsilon}{\delta} \bar{\rho}^{-\frac{1+\gamma}{2}}+\bar{\rho}^{-\gamma} \bar{u}_{y}\right) \bar{\rho} \bar{u}_{y} \psi^{2} d y d \tau
$$




$$
\begin{aligned}
& \quad+C \int_{0}^{\tau} \int_{\mathbf{R}}\left(\frac{\epsilon}{\delta}\left(\bar{\rho}^{-1-2 \gamma} \bar{u}_{y}\right)^{\frac{1}{2}}+\left(\bar{\rho}^{-3 \gamma} \bar{u}_{y}^{3}\right)^{\frac{1}{2}}\right)\left|\sqrt{\bar{\rho}^{\gamma-3}} \phi_{y}\right||\sqrt{\bar{u}} \overline{\bar{\rho}} \psi| d y d \tau \\
& \quad+C \int_{0}^{\tau} \int_{\mathbf{R}} \frac{\epsilon}{\delta}\left(\bar{\rho}^{-1-2 \gamma} \bar{u}_{y}\right)^{\frac{1}{2}}\left|\sqrt{\bar{\rho}^{\gamma-3}} \phi_{y}\right|\left|\sqrt{\bar{u}_{y} \bar{\rho}^{\gamma-2}} \phi\right| d y d \tau \\
& \quad+C \int_{0}^{\tau} \int_{\mathbf{R}} \bar{\rho}^{-\gamma} \bar{u}_{y}\left|\sqrt{\bar{u}_{y} \bar{\rho}} \psi\right|\left|\sqrt{\bar{u}_{y} \bar{\rho}^{\gamma-2}} \phi\right| d y d \tau \\
& \leq \frac{1}{8} \int_{0}^{\tau} \int_{\mathbf{R}}^{\bar{\rho}^{\gamma-3}} \phi_{y}^{2} d y d \tau+C \frac{\epsilon}{\mu^{\gamma} \delta} \int_{0}^{\tau} \int_{\mathbf{R}} \bar{u}_{y}\left(\bar{\rho}^{\gamma-2} \phi^{2}+\bar{\rho} \psi^{2}\right) d y d \tau \\
& \leq \frac{1}{8} \int_{0}^{\tau} \int_{\mathbf{R}}^{\bar{\rho}^{\gamma-3} \phi_{y}^{2} d y d \tau+C \epsilon^{(1 / 2-\gamma a)}|\ln \epsilon|^{1 / 2-\gamma} .}
\end{aligned}
$$

Finally, one has

$$
J_{3} \leq \frac{1}{8} \int_{0}^{\tau} \int_{\mathbf{R}} \bar{\rho}^{\gamma-3} \phi_{y}^{2} d y d \tau+C \int_{0}^{\tau} \int_{\mathbf{R}} \bar{\rho}^{-1-\gamma} g^{2} d y d \tau
$$

Recalling (3.8), (3.13), and Lemma 2.3, one can get

$$
\begin{aligned}
|g| & \leq\left|\bar{u}_{y y}\right|+\left|\bar{\rho} \bar{u}_{y} \psi\right|+C\left(\left|\bar{\rho}_{y} \zeta\right|+\left|\bar{\rho}_{y} \bar{\rho}^{\gamma-2} \phi\right|\right) \\
& \leq\left|\bar{u}_{y y}\right|+C \bar{u}_{y}\left(|\bar{\rho} \psi|+\left|\bar{\rho}^{\frac{3-\gamma}{2}} \zeta\right|+\left|\bar{\rho}^{\frac{\gamma-1}{2}} \phi\right|\right) .
\end{aligned}
$$

Thus the last term in (3.36) can be estimated by

$$
\begin{aligned}
& \int_{0}^{\tau} \int_{\mathbf{R}} \bar{\rho}^{-1-\gamma} g^{2} d y d \tau \\
\leq & C \int_{0}^{\tau} \int_{\mathbf{R}} \frac{\bar{u}_{y y}^{2}}{\bar{\rho}^{1+\gamma}} d y d \tau+C \int_{0}^{\tau} \int_{\mathbf{R}} \bar{\rho}^{-\gamma} \bar{u}_{y}^{2}\left(\bar{\rho}^{\gamma-2} \phi^{2}+\bar{\rho} \psi^{2}+\bar{\rho}^{2-\gamma} \zeta^{2}\right) d y d \tau \\
\leq & C \mu^{-1-\gamma} \int_{0}^{\tau}\left\|\bar{u}_{y y}\right\|^{2} d \tau+C \frac{\epsilon}{\mu^{\gamma} \delta} \int_{0}^{\tau} \int_{\mathbf{R}} \bar{u}_{y}\left(\bar{\rho}^{\gamma-2} \phi^{2}+\bar{\rho} \psi^{2}+\bar{\rho}^{2-\gamma} \zeta^{2}\right) d y d \tau \\
\leq & C \mu^{-1-\gamma}\left(\frac{\epsilon}{\delta}\right)^{2}+\frac{1}{8} \int_{0}^{\tau} \int_{\mathbf{R}} \bar{u}_{y}\left(\bar{\rho}^{\gamma-2} \phi^{2}+\bar{\rho} \psi^{2}+\bar{\rho}^{2-\gamma} \zeta^{2}\right) d y d \tau \\
\leq & C \epsilon^{(1 / 2-\gamma a)}|\ln \epsilon|^{1 / 2-\gamma} .
\end{aligned}
$$

Substituting (3.34)-(3.36) and (3.38) into (3.33) and recalling (3.12), it holds that

$$
\begin{aligned}
& \int_{\mathbf{R}}\left(\bar{\rho}^{\gamma-2} \phi^{2}+\bar{\rho} \psi^{2}+\bar{\rho}^{2-\gamma} \zeta^{2}+\frac{\phi_{y}^{2}}{\bar{\rho}^{3}}\right) d y \\
& \quad+\int_{0}^{\tau} \int_{\mathbf{R}}\left[\psi_{y}^{2}+\bar{\rho}^{1-\gamma} \zeta_{y}^{2}+\bar{\rho}^{\gamma-3} \phi_{y}^{2}+\left(\bar{\rho}^{\gamma-2} \phi^{2}+\bar{\rho} \psi^{2}+\bar{\rho}^{2-\gamma} \zeta^{2}\right) \bar{u}_{y}\right] d y d \tau \\
& \leq C \epsilon^{(1 / 2-\gamma a)}|\ln \epsilon|^{1 / 2-\gamma} \quad \text { if } \quad \epsilon \ll 1 .
\end{aligned}
$$

Step 3. Finally, we estimate $\sup _{\tau}\left\|\psi_{y}\right\|$ and $\sup _{\tau}\left\|\zeta_{y}\right\|$. For this, firstly multiplying (3.5) by $-\psi_{y y} / \rho$ gives

$$
\left(\frac{\psi_{y}^{2}}{2}\right)_{\tau}-\left(\psi_{y} \psi_{\tau}+u \frac{\psi_{y}^{2}}{2}\right)_{y}+\bar{u}_{y} \frac{\psi_{y}^{2}}{2}+\frac{\psi_{y y}^{2}}{\rho}=g \frac{\psi_{y y}}{\rho}-\frac{\psi_{y}^{3}}{2}+(\gamma-1)\left(\theta \phi_{y}+\rho \zeta_{y}\right) \frac{\psi_{y y}}{\rho} .
$$


Then integrating the above equation over $\mathbf{R}^{1} \times[0, \tau]$ yields

$$
\begin{aligned}
& \int_{\mathbf{R}} \psi_{y}^{2} d y+\int_{0}^{\tau} \int_{\mathbf{R}}\left(\bar{u}_{y} \psi_{y}^{2}+\frac{\psi_{y y}^{2}}{\bar{\rho}}\right) d y d \tau \\
= & \int_{0}^{\tau} \int_{\mathbf{R}}\left(g \frac{\psi_{y y}}{\rho}-\frac{\psi_{y}^{3}}{2}+(\gamma-1)\left(\theta \phi_{y}+\rho \zeta_{y}\right) \frac{\psi_{y y}}{\rho}\right) d y d \tau .
\end{aligned}
$$

First, one has

$$
\begin{aligned}
& \left|\int_{0}^{\tau} \int_{\mathbf{R}}(\gamma-1)\left(\theta \phi_{y}+\rho \zeta_{y}\right) \frac{\psi_{y y}}{\rho} d y d \tau\right| \\
\leq & \frac{1}{8} \int_{0}^{\tau} \int_{\mathbf{R}} \frac{\psi_{y y}^{2}}{\bar{\rho}} d y d \tau+C \int_{0}^{\tau} \int_{\mathbf{R}} \bar{\rho}^{\gamma}\left(\bar{\rho}^{\gamma-3} \phi_{y}^{2}+\frac{\zeta_{y}^{2}}{\bar{\theta}}\right) d y d \tau \\
\leq & \frac{1}{8} \int_{0}^{\tau} \int_{\mathbf{R}} \frac{\psi_{y y}^{2}}{\bar{\rho}} d y d \tau+C \epsilon^{(1 / 2-\gamma a)}|\ln \epsilon|^{1 / 2-\gamma} .
\end{aligned}
$$

Then it follows from (3.38) that

$$
\begin{aligned}
\left|\int_{0}^{\tau} \int_{\mathbf{R}} g \frac{\psi_{y y}}{\rho} d y d \tau\right| & \leq \frac{1}{8} \int_{0}^{\tau} \int_{\mathbf{R}} \frac{\psi_{y y}^{2}}{\bar{\rho}}+C \int_{0}^{\tau} \int_{\mathbf{R}} \bar{\rho}^{-1} g^{2} d y d \tau \\
& \leq \frac{1}{8} \int_{0}^{\tau} \int_{\mathbf{R}} \frac{\psi_{y y}^{2}}{\bar{\rho}}+C \epsilon^{(1 / 2-\gamma a)}|\ln \epsilon|^{1 / 2-\gamma} .
\end{aligned}
$$

Furthermore, we can compute that

$$
\begin{aligned}
\left|\int_{0}^{\tau} \int_{\mathbf{R}} \frac{\psi_{y}^{3}}{2} d y d \tau\right| & \leq C \int_{0}^{\tau}\left\|\psi_{y y}\right\|^{\frac{1}{2}}\left\|\psi_{y}\right\|^{\frac{5}{2}} d \tau \\
& \leq \frac{1}{8} \int_{0}^{\tau} \int_{\mathbf{R}} \frac{\psi_{y y}^{2}}{\bar{\rho}} d y d \tau+C \int_{0}^{\tau}\left\|\psi_{y}\right\|^{\frac{10}{3}} d \tau \\
& \leq \frac{1}{8} \int_{0}^{\tau} \int_{\mathbf{R}} \frac{\psi_{y y}^{2}}{\bar{\rho}} d y d \tau+C \sup _{\tau \in\left[0, \tau_{1}\right]}\left\|\psi_{y}\right\|^{\frac{4}{3}} \int_{0}^{\tau}\left\|\psi_{y}\right\|^{2} d \tau \\
& \leq \frac{1}{8} \int_{0}^{\tau} \int_{\mathbf{R}} \frac{\psi_{y y}^{2}}{\bar{\rho}} d y d \tau+C \int_{0}^{\tau}\left\|\psi_{y}\right\|^{2} d \tau,
\end{aligned}
$$

where in the last inequality we have used the a priori assumptions (3.11).

Substituting (3.42)-(3.44) into (3.41) and using (3.13) and (3.39), it holds that

$$
\int_{\mathbf{R}} \psi_{y}^{2} d y+\int_{0}^{\tau} \int_{\mathbf{R}}\left(\bar{u}_{y} \psi_{y}^{2}+\frac{\psi_{y y}^{2}}{\bar{\rho}}\right) d y d \tau \leq C \epsilon^{(1 / 2-\gamma a)}|\ln \epsilon|^{1 / 2-\gamma} .
$$

Secondly, multiplying (3.6) by $-\zeta_{y y} / \rho$ leads to

$$
\left(\frac{\zeta_{y}^{2}}{2}\right)_{\tau}-\left(\zeta_{y} \zeta_{\tau}+u \frac{\zeta_{y}^{2}}{2}\right)_{y}+\bar{u}_{y} \frac{\zeta_{y}^{2}}{2}+\frac{\zeta_{y y}^{2}}{\rho}=h \frac{\zeta_{y y}}{\rho}-\frac{\psi_{y} \zeta_{y}^{2}}{2} .
$$

Integrating the above equation over $\mathbf{R}^{1} \times[0, \tau]$ yields

$$
\int_{\mathbf{R}} \frac{\zeta_{y}^{2}}{2} d y+\int_{0}^{\tau} \int_{\mathbf{R}}\left(\frac{\bar{u}_{y} \zeta_{y}^{2}}{2}+\frac{\zeta_{y y}^{2}}{\rho}\right) d y d \tau=\int_{0}^{\tau} \int_{\mathbf{R}}\left(h \frac{\zeta_{y y}}{\rho}-\frac{\psi_{y} \zeta_{y}^{2}}{2}\right) d y d \tau .
$$


By Sobolev's inequality and (3.45), we can get

$$
\begin{aligned}
\left|\int_{0}^{\tau} \int_{\mathbf{R}} \frac{\psi_{y} \zeta_{y}^{2}}{2} d y d \tau\right| & \leq C \int_{0}^{\tau}\left\|\psi_{y}\right\|^{\frac{1}{2}}\left\|\psi_{y y}\right\|^{\frac{1}{2}}\left\|\zeta_{y}\right\|^{2} d \tau \\
& \leq \frac{1}{8} \int_{0}^{\tau} \int_{\mathbf{R}} \frac{\psi_{y y}^{2}}{\bar{\rho}} d y d \tau+C \int_{0}^{\tau}\left\|\psi_{y}\right\|^{\frac{2}{3}}\left\|\zeta_{y}\right\|^{\frac{8}{3}} d \tau \\
& \leq \frac{1}{8} \int_{0}^{\tau} \int_{\mathbf{R}} \frac{\psi_{y y}^{2}}{\bar{\rho}} d y d \tau+C \sup _{\left[0, \tau_{1}(\epsilon)\right]}\left\|\psi_{y}\right\|^{\frac{2}{3}}\left\|\zeta_{y}\right\|^{\frac{2}{3}} \int_{0}^{\tau} \int_{\mathbf{R}} \frac{\zeta_{y}^{2}}{\bar{\theta}} d y d \tau \\
& \leq \frac{1}{8} \int_{0}^{\tau} \int_{\mathbf{R}} \frac{\psi_{y y}^{2}}{\bar{\rho}} d y d \tau+C \int_{0}^{\tau} \int_{\mathbf{R}} \frac{\zeta_{y}^{2}}{\bar{\theta}} d y d \tau,
\end{aligned}
$$

where in the last inequality we have used the a priori assumptions (3.11). Recalling (3.8), (3.13), and Lemma 2.3, one can get

$$
|h| \leq C\left\{\left(\left|\bar{\theta}_{y y}\right|+\bar{u}_{y}^{2}\right)+\bar{u}_{y}\left(|\bar{\rho} \zeta|+\left|\psi_{y}\right|+\left|\bar{\rho} \bar{\theta}^{\frac{1}{2}} \psi\right|\right)+\left|\bar{\rho} \bar{\theta} \psi_{y}\right|+\psi_{y}^{2}\right\} .
$$

By Cauchy's inequality, it holds that

$$
\begin{aligned}
\left|\int_{0}^{\tau} \int_{\mathbf{R}} h \frac{\zeta_{y y}}{\rho} d y d \tau\right| & \leq \frac{1}{8} \int_{0}^{\tau} \int_{\mathbf{R}} \frac{\zeta_{y y}^{2}}{\bar{\rho}} d y d \tau+C \int_{\mathbf{R}} \frac{h^{2}}{\bar{\rho}} d y d \tau \\
& :=\frac{1}{8} \int_{0}^{\tau} \int_{\mathbf{R}} \frac{\zeta_{y y}^{2}}{\bar{\rho}} d y d \tau+\sum_{i=1}^{4} L_{i} .
\end{aligned}
$$

Now we estimate the terms on the right-hand side of (3.50) one by one. By Lemma 2.3 , one has

$$
\begin{aligned}
L_{1} & =\int_{0}^{\tau} \int_{\mathbf{R}} \bar{\rho}^{-1}\left(\left|\bar{\theta}_{y y}\right|^{2}+\bar{u}_{y}^{4}\right) d y d \tau \\
& \leq C \mu^{-1} \int_{0}^{\tau}\left(\left\|\bar{u}_{y}\right\|_{L^{4}}^{4}+\left\|\bar{u}_{y y}\right\|^{2}\right) d \tau \leq C \mu^{-1}\left(\frac{\epsilon}{\delta}\right)^{2} .
\end{aligned}
$$

Similarly,

$$
\begin{aligned}
L_{2} & \leq C \int_{0}^{\tau} \int_{\mathbf{R}} \bar{\rho}^{-1} \bar{u}_{y}^{2}\left(\bar{\rho}^{2} \zeta^{2}+\psi_{y}^{2}+\bar{\rho}^{2} \bar{\theta} \psi^{2}\right) d y d \tau \\
& \leq C \mu^{-1}\left(\frac{\epsilon}{\delta}\right)^{2} \int_{0}^{\tau} \int_{\mathbf{R}} \psi_{y}^{2} d y d \tau+C \int_{0}^{\tau} \int_{\mathbf{R}} \bar{\rho}^{\gamma-1} \bar{u}_{y}^{2}\left(\bar{\rho}^{2-\gamma} \zeta^{2}+\bar{\rho} \psi^{2}\right) d y d \tau \\
& \leq C \mu^{-1}\left(\frac{\epsilon}{\delta}\right)^{2} \int_{0}^{\tau} \int_{\mathbf{R}} \psi_{y}^{2} d y d \tau+C \frac{\epsilon}{\delta} \int_{0}^{\tau} \int_{\mathbf{R}} \bar{u}_{y}\left(\bar{\rho}^{2-\gamma} \zeta^{2}+\bar{\rho} \psi^{2}\right) d y d \tau
\end{aligned}
$$

and

$$
L_{3}=\int_{0}^{\tau} \int_{\mathbf{R}} \bar{\rho}^{-1} \bar{\rho}^{2} \bar{\theta}^{2} \psi_{y}^{2} d y d \tau \leq C \int_{0}^{\tau} \int_{\mathbf{R}} \psi_{y}^{2} d y d \tau
$$


By Sobolev's inequality and recalling (3.45), we can get

$$
\begin{aligned}
L_{4} & =\int_{0}^{\tau} \int_{\mathbf{R}} \frac{\psi_{y}^{4}}{\bar{\rho}} d y d \tau \leq C \mu^{-1} \int_{0}^{\tau}\left\|\psi_{y}\right\|^{3}\left\|\psi_{y y}\right\| d \tau \\
& \leq \frac{1}{8} \int_{0}^{\tau} \int_{\mathbf{R}} \frac{\psi_{y y}^{2}}{\bar{\rho}} d y d \tau+C \mu^{-2} \int_{0}^{\tau}\left\|\psi_{y}\right\|^{6} d \tau \\
& \leq C \epsilon^{(1 / 2-\gamma a)}|\ln \epsilon|^{1 / 2-\gamma}+C \mu^{-2} \sup _{\left[0, \tau_{1}(\epsilon)\right]}\left\|\psi_{y}\right\|^{4} \int_{0}^{\tau} \int_{\mathbf{R}} \psi_{y}^{2} d y d \tau \\
& \leq C \epsilon^{(1 / 2-\gamma a)}|\ln \epsilon|^{1 / 2-\gamma}+C \epsilon^{1-2 a(\gamma+1)}|\ln \epsilon|^{-1-2 \gamma} \int_{0}^{\tau} \int_{\mathbf{R}} \psi_{y}^{2} d y d \tau \\
& \leq C \epsilon^{(1 / 2-\gamma a)}|\ln \epsilon|^{1 / 2-\gamma},
\end{aligned}
$$

where in the last inequality we have used (3.45) and the fact that

$$
\epsilon^{1-2 a(\gamma+1)}|\ln \epsilon|^{-1-2 \gamma} \leq 1, \quad \text { if } \epsilon \ll 1 .
$$

Substituting (3.48) and (3.50)-(3.54) into (3.47), we can get

$$
\int_{\mathbf{R}} \frac{\zeta_{y}^{2}}{2} d y+\int_{0}^{\tau} \int_{\mathbf{R}}\left(\frac{\bar{u}_{y} \zeta_{y}^{2}}{2}+\frac{\zeta_{y y}^{2}}{\rho}\right) d y d \tau \leq C \epsilon^{(1 / 2-\gamma a)}|\ln \epsilon|^{1 / 2-\gamma}
$$

Therefore, (3.14) can be derived directly from (3.39), (3.45), and (3.55). It follows from (3.14) that if $1<\gamma \leq 2$, then

$$
\begin{aligned}
\sup _{0 \leq \tau \leq \tau_{1}(\epsilon)}\|\phi(\cdot, \tau)\|_{L^{\infty}} & \leq \sqrt{2} \sup _{0 \leq \tau \leq \tau_{1}(\epsilon)}\|\phi(\cdot, \tau)\|^{1 / 2}\left\|\phi_{y}(\cdot, \tau)\right\|^{1 / 2} \\
& \leq C \sup _{0 \leq \tau \leq \tau_{1}(\epsilon)}\left(\int_{\mathbf{R}} \bar{\rho}^{\gamma-2} \phi^{2} d y\right)^{\frac{1}{4}}\left(\int_{\mathbf{R}} \bar{\rho}^{-3} \phi_{y}^{2} d y\right)^{\frac{1}{4}} \\
& =C \epsilon^{\frac{1}{2(\gamma+2)}}|\ln \epsilon|^{1 / 4-\gamma / 2}
\end{aligned}
$$

and

$$
\begin{aligned}
\sup _{0 \leq \tau \leq \tau_{1}(\epsilon)}\|\zeta(\cdot, \tau)\|_{L^{\infty}} & \leq \sqrt{2} \sup _{0 \leq \tau \leq \tau_{1}(\epsilon)}\|\zeta(\cdot, \tau)\|^{1 / 2}\left\|\zeta_{y}(\cdot, \tau)\right\|^{1 / 2} \\
& \leq C \mu^{\frac{\gamma-2}{4}} \sup _{0 \leq \tau \leq \tau_{1}(\epsilon)}\left(\int_{\mathbf{R}} \bar{\rho}^{2-\gamma} \zeta^{2} d y\right)^{\frac{1}{4}}\left(\int_{\mathbf{R}} \zeta_{y}^{2} d y\right)^{\frac{1}{4}} \\
& =C \epsilon^{\frac{1}{8}}|\ln \epsilon|^{-\frac{1+\gamma}{4}} .
\end{aligned}
$$

If $\gamma>2$, then

$$
\begin{aligned}
\sup _{0 \leq \tau \leq \tau_{1}(\epsilon)}\|\phi(\cdot, \tau)\|_{L^{\infty}} & \leq \sqrt{2} \sup _{0 \leq \tau \leq \tau_{1}(\epsilon)}\|\phi(\cdot, \tau)\|^{1 / 2}\left\|\phi_{y}(\cdot, \tau)\right\|^{1 / 2} \\
& \leq C \mu^{\frac{2-\gamma}{4}} \sup _{0 \leq \tau \leq \tau_{1}(\epsilon)}\left(\int_{\mathbf{R}} \bar{\rho}^{\gamma-2} \phi^{2} d y\right)^{\frac{1}{4}}\left(\int_{\mathbf{R}} \bar{\rho}^{-3} \phi_{y}^{2} d y\right)^{\frac{1}{4}} \\
& =C \epsilon^{\frac{1}{8}}|\ln \epsilon|^{\frac{3(1-\gamma)}{4}}
\end{aligned}
$$

and

$$
\begin{aligned}
\sup _{0 \leq \tau \leq \tau_{1}(\epsilon)}\|\zeta(\cdot, \tau)\|_{L^{\infty}} & \leq \sqrt{2} \sup _{0 \leq \tau \leq \tau_{1}(\epsilon)}\|\zeta(\cdot, \tau)\|^{1 / 2}\left\|\zeta_{y}(\cdot, \tau)\right\|^{1 / 2} \\
& \leq C \sup _{\substack{0 \leq \tau \leq \tau_{1}(\epsilon) \\
\text { 年 }}}\left(\int_{\mathbf{R}} \bar{\rho}^{2-\gamma} \zeta^{2} d y\right)^{\frac{1}{4}}\left(\int_{\mathbf{R}} \zeta_{y}^{2} d y\right)^{\frac{1}{4}} \\
& =C \epsilon^{\frac{\gamma(3 \gamma-2)}{2(3 \gamma-2)}}|\ln \epsilon|^{\frac{1-2 \gamma}{4}}
\end{aligned}
$$


Meanwhile we also have

$$
\sup _{\tau \in\left[0, \tau_{1}(\epsilon)\right]}\left(\left\|\psi_{y}\right\|+\left\|\zeta_{y}\right\|\right) \leq C \epsilon^{\left(\frac{1}{4}-\frac{\gamma a}{2}\right)}|\ln \epsilon|^{1 / 4-\gamma / 2} \leq 1 .
$$

So the a priori assumptions (3.11) are verified if $\epsilon \ll 1$. The proof of Lemma 3.2 is completed.

Note that the obtained a priori estimates (3.56)-(3.60) are better than the a priori assumptions (3.11) in the maximum time interval $\left[0, \tau_{1}(\epsilon)\right]$. Based on these a priori estimates, we can claim $\tau_{1}(\epsilon)=\infty$. If $\tau_{1}(\epsilon)<\infty$, then by again using the local existence at time $\tau=\tau_{1}(\epsilon)$, we can find anther time $\tau_{2}(\epsilon)>\tau_{1}(\epsilon)$ so that the solution satisfies the assumptions (3.11) in the time interval $\left[0, \tau_{2}(\epsilon)\right]$ which contradicts the assumption that $\tau_{1}(\epsilon)$ is the maximum time. Therefore we extend the local solution to the global solution in $[0, \infty)$ for small but fixed $\epsilon$.

Proof. (Proof of Theorem 1.1) It remains to prove (1.14) with $a, b$, and $c$ given in (1.15)-(1.17) respectively. From Lemma 2.2, Lemma 2.3 (iii), Lemma 3.2, and recalling that $\mu=\epsilon^{a}|\ln \epsilon|$ and $\delta=\epsilon^{a}$, it holds that for any given positive constant $l$ there exists a constant $C_{l}>0$ which is independent of $\epsilon$ such that

$$
\begin{aligned}
& \sup _{t \geq l}\left\|\rho(\cdot, t)-\rho^{r_{3}}(\dot{\bar{t}})\right\|_{L^{\infty}} \\
& \leq \sup _{\tau \in[0,+\infty)}\|\phi(\cdot, \tau)\|_{L^{\infty}}+\sup _{t \geq l}\left\|\bar{\rho}(\cdot, t)-\rho_{\mu}^{r_{3}}\left(\frac{\cdot}{t}\right)\right\|_{L^{\infty}}+\sup _{t \geq l}\left\|\rho_{\mu}^{r_{3}}\left(\frac{\dot{\bar{t}}}{t}\right)-\rho^{r_{3}}\left(\frac{\dot{\bar{t}}}{t}\right)\right\|_{L^{\infty}} \\
& \leq\left\{\begin{array}{lll}
C_{l}\left(\epsilon^{\frac{1}{2(\gamma+2)}}|\ln \epsilon|^{1 / 4-\gamma / 2}+\delta|\ln \delta|+\mu\right), & \text { if } & 1<\gamma \leq 2, \\
C_{l}\left(\epsilon^{\frac{1}{8}}|\ln \epsilon|^{3(1-\gamma) / 4}+\delta|\ln \delta|+\mu\right), & \text { if } \quad & \gamma>2,
\end{array}\right. \\
& \leq C_{l} \epsilon^{a}|\ln \epsilon|, \\
& \sup _{t \geq l}\left\|m(\cdot, t)-m^{r_{3}}\left(\frac{\cdot}{\bar{t}}\right)\right\|_{L^{\infty}} \\
& \leq \sup _{t \geq l}\left(\|m(\cdot, t)-\bar{m}(\cdot, t)\|_{L^{\infty}}+\left\|\bar{m}(\cdot, t)-m_{\mu}^{r_{3}}(\dot{\bar{t}})\right\|_{L^{\infty}}+\left\|m_{\mu}^{r_{3}}(\dot{\bar{t}})-m^{r_{3}}(\dot{\bar{t}})\right\|_{L^{\infty}}\right) \\
& \leq C \sup _{\tau \in[0,+\infty)}\left(\|\psi\|_{L^{\infty}}+\|\phi\|_{L^{\infty}}\right)+\sup _{t \geq l}\left\|\bar{m}(\cdot, t)-m_{\mu}^{r_{3}}(\dot{\bar{t}})\right\|_{L^{\infty}} \\
& +\sup _{t \geq l}\left\|m_{\mu}^{r_{3}}(\dot{\bar{t}})-m^{r_{3}}(\dot{\bar{t}})\right\|_{L^{\infty}} \\
& \leq C_{l} \epsilon^{b}|\ln \epsilon| \text {, }
\end{aligned}
$$

and

$$
\begin{aligned}
& \quad \sup _{t \geq l}\left\|\widetilde{e}(\cdot, t)-\widetilde{e^{r_{3}}}\left(\frac{\dot{t}}{t}\right)\right\|_{L^{\infty}} \\
& \leq C \sup _{\tau \in[0,+\infty)}\left(\|\zeta(\cdot, \tau)\|_{L^{\infty}}+\|\phi(\cdot, \tau)\|_{L^{\infty}}\right)+\sup _{t \geq l}\left\|\bar{\rho} \bar{\theta}(\cdot, t)-\widetilde{e_{\mu}^{r_{3}}}\left(\frac{\dot{\bar{t}}}{t}\right)\right\|_{L^{\infty}} \\
& \quad+\sup _{t \geq l}\left\|\widetilde{e_{\mu}^{r_{3}}}(\cdot \overline{\bar{t}})-\widetilde{e^{r_{3}}}\left(\frac{\dot{\bar{t}}}{t}\right)\right\|_{L^{\infty}} \\
& \leq C_{l} \epsilon^{c}|\ln \epsilon| .
\end{aligned}
$$


Thus the proof of Theorem 1.1 is completed.

Acknowledgment. The work of M.-J. Li is supported by the NSFC Grant No. 11201503, and the authors would like to thank Professors Feimin Huang and Yi Wang for the valuable comments and suggestions which greatly improved the presentation of the paper.

\section{REFERENCES}

[1] G.K. Batchelor, An Introduction to Fluid Dynamics, Cambridge University Press, 1967.

[2] G.Q. Chen and M. Perepelitsa, Vanishing viscosity limit of the Navier-Stokes equations to the Euler equations for compressible fluid flow, Commun. Pure Appl. Math., 63, 1469-1504, 2010.

[3] F.M. Huang, M.J. Li, and Y. Wang, Zero dissipation limit to rarefaction waves with vacuum for 1-D compressible isentropic Navier-Stokes equations, SIAM J. Math. Anal., 44, 1742-1759, 2012.

[4] F.M. Huang and X.H. Qin, Stability of boundary layer and rarefaction wave to an outflow problem for compressible Navier-Stokes equations under large perturbation, J. Diff. Eqs., 246, 4077-4096, 2009.

[5] F. Huang, Y. Wang, Y. Wang, and T. Yang, The limit of the Boltzmann equation to the Euler equations for Riemann problems, SIAM J. Math. Anal., 45, 1741-1811, 2013.

[6] F.M. Huang, Y. Wang, and T. Yang, Hydrodynamic limit of the Boltzmann equation with contact discontinuities, Commun. Math. Phys., 295, 293-326, 2010.

[7] F.M. Huang, Y. Wang, and T. Yang, Fluid dynamic limit to the Riemann solutions of Euler equations: I. Superposition of rarefaction waves and contact discontinuity, Kin. Rel. Models, 3, 685-728, 2010.

[8] F.M. Huang, Y. Wang, and T. Yang, Vanishing viscosity limit of the compressible NavierStokes equations for solutions to Riemann problem, Arch. Rat. Mech. Anal., 203, 379-413, 2012.

[9] S. Jiang, G.X. Ni, and W.J. Sun, Vanishing viscosity limit to rarefaction waves for the NavierStokes equations of one-dimensional compressible heat-conducting fluids, SIAM J. Math. Anal., 38, 368-384, 2006.

[10] Q.S. Jiu, Y. Wang, and Z.P. Xin, Vacuum behaviors around rarefaction waves to $1 D$ compressible Navier-Stokes equations with density-dependent viscosity, SIAM J. Math. Anal., 45, 3194-3228, 2013.

[11] M.J. Li, T. Wang, and Y. Wang, The limit to rarefaction wave with vacuum for $1 D$ compressible fluids with temperature-dependent viscosities, preprint, 2013. http://arxiv.org/abs/1307.3382

[12] T.P. Liu, and J. Smoller, On the vacuum state for the isentropic gas dynamics equations, Adv. in Appl. Math., 1, 345-359, 1980.

[13] T. Liu, T. Yang, S.H. Yu, and H.J. Zhao, Nonlinear stability of rarefaction waves for the Boltzmann equation, Arch. Rat. Mech. Anal., 181, 333-371, 2006.

[14] S.X. Ma, Zero dissipation limit to strong contact discontinuity for the 1-D compressible NavierStokes equations, J. Diff. Eqs., 248, 95-110, 2010.

[15] A. Matsumura, and K. Nishihara, Asymptotics toward the rarefaction waves of the solutions of a one-dimensional model system for compressible viscous gas, Japan J. Appl. Math., 3, $1-13,1986$.

[16] M. Perepelitsa, Asymptotics toward rarefaction waves and vacuum for 1-D compressible Navier-Stokes equations, SIAM J. Math. Anal., 42, 1404-1412, 2010.

[17] X.H. Qin, Large-time behaviour of solutions to the outflow problem of full compressible NavierStokes equations, Nonlinearity, 24, 1369-1394, 2011.

[18] J. Smoller, Shock Waves and Reaction-Diffusion Equations, Second Edition, Grundlehren der Mathematischen Wissenschaften, New York: Springer-Verlag, xxii, 258, 1994.

[19] Y. Wang, Zero dissipation limit of the compressible heat-conducting Navier-Stokes equations in the presence of the shock, Acta Math. Sci., 28B, 727-748, 2008.

[20] Z.P. Xin, Zero dissipation limit to rarefaction waves for the one-dimensional Navier-Stokes equations of compressible isentropic gases, Commun. Pure Appl. Math., 46, 621-665, 1993.

[21] Z.P. Xin and H.H. Zeng, Convergence to the rarefaction waves for the nonlinear Boltzmann equation and compressible Navier-Stokes equations, J. Diff. Eqs., 249, 827-871. 2010. 